\title{
Widerstand oder Terrorismus? Eine theoretische Grundlegung
}

\author{
Frauke Höntzsch ii
}

Eingegangen: 4. Dezember 2019 / Überarbeitet: 20. November 2020 / Angenommen: 3. Dezember 2020 / Online publiziert: 7. Januar 2021

(C) Der/die Autor(en) 2020

Zusammenfassung Terrorismus ist ein nicht nur politisch, sondern auch politikwissenschaftlich umstrittener Begriff. Das liegt in dem Umstand begründet, dass mit der Kennzeichnung politischer Gewalt als Terrorismus eine Wertung verbunden ist: Terrorismus gilt als illegitime Gewalt - im Gegensatz zu Widerstand, der gleichfalls illegal ist, aber als legitim verstanden wird. Die gängigen wissenschaftlichen Terrorismusdefinitionen versuchen diese Wertung auszublenden und definieren Terrorismus als Taktik und damit als Mittel zum Zweck; der Analyse bzw. Auswahl empirischer Phänomene aber geht eine Wertung notwendig voraus. Der vorliegende Beitrag schlägt demgegenüber eine Konzeption von Terrorismus vor, die die Ziele und damit die Frage nach der Legitimation politischer Gewalt systematisch miteinbezieht. Die vorgeschlagene Konzeptualisierung nimmt das Selbstverständnis von Akteuren politischer Gewalt, die die von ihnen verübte Gewalt notwendig als legitim, mithin als Widerstand, begreifen müssen, zum Ausgangspunkt und misst die Berechtigung dieser Selbstzuschreibung anhand der klassischen Rechtfertigung gewaltsamen Widerstands. Der vorliegende Beitrag entwickelt auf dieser Grundlage einen mehrstufigen Maßstab zur Unterscheidung legitimer und illegitimer politischer Gewalt, der von den moralischen Überzeugungen der Akteure abstrahiert.

Schlüsselwörter Politische Gewalt · Legitimation · Delegitimation · Befreiung · Revolution 


\section{Resistance or Terrorism? A Theoretical Foundation}

Abstract Terrorism is a controversial term, not only in politics but also in political science. The reason for this controversy seems to be that labeling political violence as terrorism is connected with a value judgment: Terrorism is considered illegitimate violence, as opposed to resistance, which is also illegal but understood to be legitimate at the same time. The common academic definitions of terrorism try to avoid such valuation and define terrorism as a tactic and thus as a means to an end, but the analysis or selection of the empirical phenomena is necessarily preceded by a valuation. In contrast, this article develops a conception of terrorism that systematically includes the goals and thus the question of legitimation of political violence. The proposed conceptualization takes as a starting point the selfconcept of the actors of political violence, who necessarily regard the violence they perpetrate as legitimate and thus as resistance, and measures the justification of this self-ascription by the classic justification of violent resistance. On this basis, this article develops a multilevel standard for distinguishing legitimate and illegitimate political violence, abstracting from the moral convictions of the actors.

Keywords Political violence $\cdot$ Legitimation · Delegitimation · Liberation · Revolution

\section{Einleitung}

Die Bezeichnung politischer Gewalt als Terrorismus ist eine Frage der Perspektive. So konstatiert Brian Jenkins mit Blick auf den politischen Gebrauch: „Use of the term implies a moral judgment; and if one party can successfully attach the label terrorist to its opponent, then it has indirectly persuaded others to adopt its moral viewpoint. Terrorism is what the bad guys do" (Jenkins 1980, S. 1). In der Tat ist nicht nur mit dem Begriff des Terrorismus, sondern auch mit seinem - perspektivisch - positiven Gegenbegriff, dem Begriff des (gewaltsamen) Widerstands, ${ }^{1}$ eine Wertung unauflöslich verknüpft: Es handelt sich bei der mit (gewaltsamem) Widerstand bzw. Terrorismus bezeichneten politischen Gewalt um illegale politische Gewalt, sprich Gewalt, die durch die Rechtsordnung, in deren Rahmen sie stattfindet, nicht legitimiert ist und die folglich per definitionem legitimationsbedürftig ist. Illegale politische Gewalt muss sich zu ihrer Rechtfertigung auf höheres, vorstaatliches Recht berufen. Die Begriffe des (gewaltsamen) Widerstands und des Terrorismus bewerten diese Legitimationsversuche; sie sprechen illegalen politischen Gewalthandlungen Legitimation zu oder ab: Terrorismus bezeichnet illegitime, Widerstand legitime politische Gewalt.

Die Wissenschaft reagiert auf die dem Terrorismusbegriff inhärente Wertung ausweichend. Mit Blick auf die Schwierigkeiten einer wissenschaftlichen Definition hält Christopher Daase fest: „Kaum ein politischer Begriff ist so umstritten, wie

\footnotetext{
1 Widerstand ist ein sehr viel umfassenderes Konzept als Terrorismus; dem Konzept des Terrorismus wird im Folgenden nur eine Form des Widerstands, gewaltsamer Widerstand, gegenübergestellt.
} 
der des Terrorismus. Das liegt daran, dass mit dem Begriff nicht nur ein Phänomen beschrieben, sondern es zugleich moralisch verurteilt wird. Terrorismus gilt als illegitime Gewalt - was aber legitim und illegitim ist, hat sich im Laufe der Zeit geändert und ist politisch umstritten“ (Daase 2013, S. 336; Herv. i. O.). Ob politische Gewalt als legitimer Widerstand oder aber als illegitim, mithin als Terrorismus, gekennzeichnet wird, ist so verstanden eine politische Frage, die wissenschaftlich nicht zu beantworten ist. Entsprechend defensiv fallen auch die wissenschaftlichen Definitionen aus: Als Terrorismus bezeichnete politische Gewalt wird überwiegend als Taktik bzw. Kommunikationsstrategie verstanden, der sich Akteure aus einer Position der Schwäche heraus bedienen. Das heißt Terrorismus wird als Mittel zum Zweck definiert und konzeptuell mehr oder weniger auf die Anwendung/-drohung von „Terror“ in strategischer Absicht verkürzt. Als Definition, die den kleinsten gemeinsamen Nenner der vorhandenen akademischen Definitionen abbildet, formulieren Weinberg et al. (2004, S. 782): „,Terrorism is a politically motivated tactic involving the threat or use of force or violence in which the pursuit of publicity plays a significant role“. Zwar besteht Uneinigkeit über die spezifischen Kennzeichen dieser als Terrorismus bezeichneten, zu politischen Zwecken eingesetzten Gewalt; so kommt Alex P. Schmid, der in einer Analyse aus dem Jahr 1984 anhand 109 Definitionen die Häufigkeit der Definitionsmerkmale des Terrorismus herausgearbeitet hat, zu dem Ergebnis, dass „Gewalt“ das einzige Charakteristikum ist, auf das sich eine klare Mehrheit (83,5\%) einigen kann, gefolgt von ,,politische“ (65\%), das dritte Merkmal „Furcht, Terror betonend“ erreicht noch $51 \%$; alle folgenden 19 Merkmale unter $50 \%$ (zitiert nach Schmid 2013, S. 74); ${ }^{2}$ doch ist den gängigen Definitionen eines gemeinsam: Sie versuchen, indem sie die politischen Ziele terroristischer Gruppen ausblenden - sie den Akteuren im Fall des ,neuen Terrorismus“ gar teils gänzlich absprechen und die ausgeübte Gewalt als Selbstzweck kennzeichnen (vgl. Münkler 2004) - erklärtermaßen, die Frage nach der Legitimation zu umgehen: „Indem man terroristische Organisationen nach diesen Vorgaben konzeptualisiert, schließt man normative oder bewertende Überlegungen von der analytischen Eingrenzung dieses Phänomens aus. Der Schwerpunkt liegt stattdessen auf der typischen Vorgehensweisen“ (Reinares 2002, S. 391).

Die dem Begriff inhärente Wertung auszuschließen, aber ist schlicht nicht möglich; sie geht auch den gängigen Definitionen notwendig voraus bzw. ist ihnen eingeschrieben, wenn diese (nach welchen Maßstäben auch immer) als Terrorismus bezeichnete Phänomene politischer Gewalt auf ihre Besonderheiten analysieren und anschließend anhand der Ergebnisse dieser Analyse zu definieren versuchen. Das heißt: Die gängigen Definitionen können die Wertung nicht vermeiden, verfügen

\footnotetext{
2 Schmid und Jongman haben daran anschließend folgende „Academic Consensus Definition“ formuliert: „Terrorism is an anxiety-inspiring method of repeated violent action, employed by (semi-) clandestine individual, group, or state actors, for idiosyncratic, criminal, or political reasons, whereby - in contrast to assassination - the direct targets of violence are not the main targets. The immediate human victims of violence are generally chosen randomly (targets of opportunity) or selectively (representative or symbolic targets) from a target population, and serve as message generators. Threat- and violence-based communication processes between terrorist (organization), (imperiled) victims, and main targets are used to manipulate the main target (audience(s)), turning it into a target of terror, a target of demands, or a target of attention, depending on whether intimidation, coercion, or propaganda is primarily sought“" (1988, S. 28).
} 
aber selbst über keinen wissenschaftlichen Maßstab, diese vorzunehmen. Eine von den jeweiligen moralischen Überzeugungen unabhängige, wissenschaftliche Kennzeichnung politischer Gewalt als illegitim kann auf Grundlage einer Analyse der empirisch beobachtbaren Vorgehensweise bzw. verwendeten Mittel nicht erfolgen. Ebenso wenig lassen sich auf dieser Grundlage Terrorismus und gewaltsamer Widerstand als illegitime und legitime politische Gewalt unterscheiden, müsste eine solche Unterscheidung doch mit zwei unterschiedlichen Parametern - Mitteln und Zielen operieren, was schon aus logischen Gründen wenig überzeugt, aber auch empirisch an seine Grenzen gerät, wo sich Widerstandsgruppen terroristischer Vorgehensweisen bedienen bzw. „Terroristen“ erklärtermaßen gegen eine als illegitim erachtete Herrschaft opponieren. ${ }^{3}$ Der Grund, dass nach wie vor gilt, was Walter Laqueur mit Blick auf eine mögliche Terrorismusdefinition bereits 1986 festhielt - „Agreement on a definition, alas, does not exist, and there is no reason to assume that it will in the foreseeable future" (Laqueur 1986, S. 88) ${ }^{4}$ - scheint folglich weniger, wie das bei Daase anklingt, in der mit dem Begriff verbundenen Wertung selbst zu liegen, als vielmehr in deren bewussten Ausblendung und damit der Ausblendung der Ziele politischer Gewalt durch die gängigen wissenschaftlichen Terror(ismus)definitionen.

Entsprechend existieren auch, trotz der in der Perspektivität der Begriffe des gewaltsamen Widerstands und des Terrorismus impliziten partiellen Überschneidung der so bezeichneten empirischen Phänomene, die auch in dem ,häufig kolportierte[n] Diktum ,des einen Terroristen, des anderen Freiheitskämpfer“"“5 (Schneckener 2015, S. 31) zum Ausdruck kommt, zwar Analysen, die den Radikalisierungsverlauf einzelner Gruppierungen nachzeichnen, ${ }^{6}$ wodurch diese sich auf einem Kontinuum

\footnotetext{
3 Das gilt auch für Ansätze, die die Frage nach der Legitimation politischer Gewalt miteinbeziehen, diese aber an der Qualität der verwendeten Mittel festzumachen versuchen. So nennt etwa Martha Crenshaw (1983) drei Legitimitätskriterien für Gewaltanwendung, die Freiheitskämpfer bzw. Guerillakämpfer ihres Erachtens erfüllen, während Terroristen mindestens eines verfehlten; Freiheitskämpfer wenden demnach Gewalt im Rahmen des Völkerrechts an, die Gewalt richtet sich nur gegen Kombattanten und die Anwendung der Gewalt hat Aussicht auf Erfolg. Ähnlich bemerkt Christopher J. Finlay (2015), ,that terrorism refers to something that some otherwise legitimate armed movements use but not others (as well as being a feature of other groups that cannot claim to be engaged in legitimate resistance)“ (S. 4) und reduziert den Begriff zugleich auf eine instrumentelle Bedeutung als ,a range of different types of indiscriminate violence" (S. 5).

${ }^{4}$ Diesen Befund bestätigend hat Alex P. Schmid im Routledge Handbook of Terrorism Research bis 2013 über 250 verschiedene Definitionen von „Terrorismus“ zusammengetragen.

5 Diese Formulierung geht auf den 40. US-Präsidenten Ronald Reagan zurück.

6 So zeigt die Forschung zur Dynamik sozialen Konflikts, ,dass den gewaltsamen Aktionen meist eine friedliche kollektive Mobilisierung vorangeht. So stellen etliche Untersuchungen eine Beziehung zwischen sozialen Bewegungen und Protestzyklen einerseits und der Gründung von terroristischen Vereinigungen andererseits her“ (Reinares 2002, S. 394). Ehud Sprinzak etwa kommt zu dem Schluss: „Rebel terrorism [...] does not emerge in a vacuum or out of an inexplicable impetus of a few crazies. Rather, it is an extension of the political world we all live in, a special case of opposition politics" (Sprinzak 1991, S. 37). Auch das Ziel von Leonard Weinberg ist es, die Verbindung zwischen Parteienpolitik und Terrorismus, verstanden als ,not an ideology but an activity“ (Weinberg 1992, S. 3), aufzuzeigen entgegen der vorherrschenden Wahrnehmung terroristischer Gewalt als ,a kind of anti-politics“ (Weinberg 1992, S. 141). Speziell mit Blick auf den ,islamistischen Terrorismus“ stellt Quintan Wiktorowicz in einem von ihm herausgegebenen Sammelband zum „Islamic Activism“ einleitend fest: „In contradistinction to popular perceptions of radical Islamic groups as irrational, ,crazy',or deviant, these groups frequently follow a particular dynamic that mirrors the rational calculus of other non-Islamic social movement actors who
} 
politischer Opposition verorten lassen, aber kaum systematische Vergleiche zwischen gewaltsamem Widerstand und Terrorismus als Formen legitimer und illegitimer Systemopposition. ${ }^{7}$ Vielmehr stellt Charles Townshend richtig fest: ,Viele, die über den Terrorismus geschrieben haben, lassen diesen Punkt außer Acht, aber wir tun gut daran, zu untersuchen, woher dieser auffällige Relativismus kommt, und zu prüfen, ob der Terrorismus wirklich eine Strategie der Befreiung sein kann“ (2005, S. 35), um dann selbst eine nur historische (mithin empirische) Antwort zu geben: „Wie die Geschichte lehrt, waren diejenigen, die eine rein terroristische Strategie verfolgten, keine erfolgreichen Befreier. Umgekehrt waren die Befreier [...] keine reinen oder absoluten Terroristen“ (Townshend 2005, S. 40-41).

Um den angesprochenen Relativismus zu überwinden, schlägt der vorliegende Beitrag in Abgrenzung zu den gängigen Definitionen eine Konzeption von Terrorismus vor, die die Frage nach den Zielen der so bezeichneten politischen Gewalt und damit die Frage nach ihrer Legitimation systematisch in den Mittelpunkt rückt. Insofern „Terrorismus“ als illegitime politische Gewalt (nicht aber das Bekenntnis zum ,Terror“) nur eine Fremdzuschreibung sein kann (weil die Akteure anderenfalls den eigenen Gewalthandlungen die Legitimation absprechen würden), ${ }^{8}$ wohingegen Widerstand zunächst als Selbstzuschreibung dienen wird (politische Gewaltakteure sich also in diesem Sinne notwendigerweise als Widerständler begreifen müssen), entwickelt der vorliegende Beitrag auf Grundlage der klassischen Rechtfertigung gewaltsamen Widerstands einen Maßstab für die normative Unterscheidung legitimer und illegitimer politischer Gewalt, der von den moralischen Überzeugungen der Gewaltakteure abstrahiert. Die vorgeschlagene Konzeptualisierung nimmt also das (in der Legitimationsbedürftigkeit illegaler politischer Gewalt angelegte) Selbstverständnis politischer Gewaltakteure als Widerständler zum Ausgangspunkt und misst dessen Berechtigung anhand einem diesem Selbstverständnis inhärenten Maßstab, nämlich anhand der Rechtfertigung gewaltsamen Widerstands durch die Befreiung von Willkürherrschaft, die, wie zu zeigen sein wird, die Forderung nach gleicher Bindung der Herrschenden und Beherrschten an gleiches Recht impliziert. Der vor-

have used violence as part of their repertoire of contention“ (Wiktorowicz 2004, S. 20). Vgl. auch Della Porta und Tarrow 1986; Della Porta 1995 und 2013.

7 Einen seltenen Versuch der Systematisierung unternimmt Birgit Enzmann (2013a), wobei die Perspektivität bzw. Wertung dem Vergleich hier eingeschrieben ist, wenn Enzmann als Ziele „Wiederherstellung der Rechtsordnung“ (Widerstand) versus „Störung der Handlungsfähigkeit des Staats“ (Terrorismus), als Adressaten „Gesamtgesellschaft“ (Widerstand) versus „Unterstützer, Staat/Regierung“ (Terrorismus) und unter Legitimität „Moral, Wertordnung“ (Widerstand) versus „Ideologie, Wertordnung“ (Terrorismus) unterscheidet (S. 47).

8 In den wenigen Fällen, in denen der Begriff „Terrorismus“ im Rahmen ideengeschichtlicher Quellen affirmativ genutzt wird, geschieht dies im Verständnis von Terrorismus als eines revolutionären Mittels, es handelt sich also um Fälle, in denen der Schwerpunkt der Argumentation nicht (oder: nicht mehr) auf der Rechtfertigung politischer Gewalt (und damit der Widerstandsterminologie) liegt, sondern auf deren mittelbarem Ziel, der Revolution - besonders prominent im Rahmen des sozialrevolutionären Terrorismus. Beispielhaft kann etwa Marx zitiert werden, der in der Neuen Rheinische Zeitung vom 7. November 1848 zum „Sieg der Konterrevolution in Wien“ schrieb: „Die resultatlosen Metzeleien seit den Juni- und Oktobertagen, das langweilige Opferfest seit Februar und März, der Kannibalismus der Kontrerevolution selbst wird die Völker überzeugen, daß es nur ein Mittel gibt, die mörderischen Todeswehen der alten Gesellschaft, die blutigen Geburtswehen der neuen Gesellschaft abzukürzen, zu vereinfachen, zu konzentrieren, nur ein Mittel - den revolutionären Terrorismus“ (Marx 1959, S. 457; Herv. i. O.). 
liegende Beitrag zielt so auf eine Kennzeichnung politischer Gewalt als Terrorismus mit wissenschaftlichen Maßstäben. Die vorgeschlagene Konzeption stellt damit gängige Terror(ismus)definitionen der Sache nach keineswegs infrage, sie versteht die Besonderheit der durch Terroristen verübten Gewalt (in Form ihrer Entgrenzung hinsichtlich Zeit, Raum, Qualität, Opfern und Zielen) jedoch als Folge fehlender Legitimation und folglich die empirische Analyse der Mittel der normativen Frage nach den Zielen nachgeordnet.

Die Konzeption des Terrorismusbegriffs über die Ziele politischer Gewalt erfolgt in ideengeschichtlicher Perspektive, indem die mit Blick auf illegale politische Gewalt maßgeblichen ideengeschichtlichen Quellen einem politikwissenschaftlichen Erkenntnisinteresse folgend auf ihre theoretischen Implikationen hin befragt werden. Anders als die überwiegend empirisch verfahrende Terrorismusforschung schließt eine solchermaßen ideengeschichtlich verfahrende Theoriebildung normative Fragen mit ein, ohne selbst eine Wertung vorzunehmen. ${ }^{9}$ Der vorliegende Beitrag erhebt folglich nicht den Anspruch, sämtliche empirische Phänomene erklären zu können, er formuliert einen systematisch-theoretischen Ordnungsvorschlag, der im besten Fall der Analyse der empirischen Phänomene als Grundlage dienen kann. Dazu soll im Folgenden zunächst die Herausbildung der klassischen Rechtfertigung von gewaltsamem (in Abgrenzung zu systeminternem) Widerstand als Maßstab für die Unterscheidung legitimer und illegitimer Gewalt aufgezeigt werden (2.). Dieser lässt sich unter Einbezug des gemeinsamen mittelbaren Ziels der als Widerstand wie der als Terrorismus bezeichneten illegalen politischen Gewalt, der Revolution, analytisch in zwei (bzw. drei) Stufen ausdifferenzieren (3.). In einem weiteren Schritt ist zu zeigen, dass die empirische und die normative Legitimation politischer Gewalt miteinander verschränkt sind, wobei die ausbleibende empirische Legitimation, die auf die mangelnde normative Legitimation verweist, empirisch in einer Entgrenzung der Gewalt zum Ausdruck kommt (4.). Plausibilisiert werden sollen die Überlegungen abschließend durch Selbstzeugnisse führender Vertreter von al-Qaida (5).

\section{Befreiung von Willkürherrschaft als Rechtfertigung gewaltsamen Widerstands}

Allen Konzeptionen des Widerstands ist dessen Kennzeichnung als Gegengewalt gemein, Widerstand reagiert demzufolge auf vorausgegangenes Unrecht. Als Aggressor gilt die illegitim errichtete, sprich qua Konstitution willkürliche, staatliche Gewalt oder aber die ursprünglich legitime, jedoch entartete und in der Folge willkürlich verfahrende staatliche Gewalt. In Anschluss an diese Unterscheidung differenzieren sich im Rahmen der Theorie des Widerstandsrechts im Laufe der Zeit zwei Formen des Widerstands heraus. Während systeminterner Widerstand (am Endpunkt der Entwicklung, im demokratischen Rechtsstaat, in Gestalt des zivilen Ungehorsams) die vorgegebene Ordnung nicht infrage stellt, zielt der hier interessierende

\footnotetext{
9 Für eine so verstandene ,politikwissenschaftliche Ideengeschichte“ und ihr spezifisches politiktheoretisches Potenzial, das ein Mittleres zwischen deskriptiver und normativer Theoriebildung bildet, vgl. Höntzsch (2015b).
} 
Widerstand gegen Willkürherrschaft auf die Beseitigung der bestehenden Ordnung und damit mittelbar auf eine Neuordnung, ist also mittelbar stets revolutionär. Weil Widerstand als Gehorsamsverweigerung bzw. Gesetzesbruch per definitionem illegal ist, kann er Legitimation über die Begründungsversuche der Akteure hinaus politisch (empirisch) erst ex post durch die politische Öffentlichkeit bzw. Mehrheit erlangen; der Theorie des Widerstandsrechts zufolge (die sich in den Begründungen der politischen Gewaltakteure widerspiegelt) gilt gewaltsamer Widerstand als legitim, wo er sich gegen eine nicht durch höheres Recht legitimierte Herrschaft richtet. Unabhängig von der jeweiligen (göttlichen oder natürlichen) Quelle des höheren Rechts beruht eine legitime Herrschaft demnach auf einem Vertrag zwischen Herrschenden und Beherrschten, der auch die Herrschenden an das höhere Recht bindet und so die Beherrschten vor Willkür schützt. Dabei erstreckt sich der Schutz vor Willkürherrschaft erst im Gesellschaftsvertragsdenken auch auf die Rechtssetzung als Auslegung der Rechtsquelle selbst, erst hier sind Herrschende und Beherrschte tatsächlich gleichermaßen gleichem Recht unterworfen und nicht einer durch die Herrschenden vorgenommenen, willkürlichen Interpretation des höheren Rechts. Der Willkürherrschaft wird hier eine machtteilige, demokratische Ordnung als Ziel des revolutionären Widerstands gegenübergestellt.

Überlegungen zum Widerstand in Form des Tyrannenmords finden sich bereits in der Antike, sowohl in der griechischen also auch in der römischen Tradition (vgl. Stüttler 1972). Während Aristoteles und Platon den Tyrannenmord nur beschreiben, sich aber eines Urteils enthalten, bezieht Cicero Stellung und rechtfertigt den Tyrannenmord mit der Verteidigung der Freiheit bzw. der rechtlich legitimierten Herrschaft. So schreibt er über Lucius Brutus, er habe ,,von seinen Mitbürgern jenes ungerechte Joch der harten Knechtschaft herabgeworfen. Obwohl er Privatmann war, hat er doch das ganze Gemeinwesen vertreten und hat als erster in diesem Staate gelehrt, daß in der Erhaltung der Freiheit der Bürger niemand Privatmann ist" (Cicero 1979, S. 213). Der Tyrannenmord ist Tat eines Einzelnen im Dienste der Gemeinschaft zum Erhalt bzw. zur Wiederherstellung der Freiheit der Bürger gegen einen Herrscher ohne Recht. Cicero formuliert in Anschluss an das griechische Verständnis eines Tyrannen: „Sobald sich nämlich dieser König zu einer ungerechten Gewaltherrschaft wendet, entsteht sogleich der Tyrann [...], der schließlich mit dem ganzen Menschengeschlecht keine Gemeinschaft des Rechtes" haben will (Cicero 1979, S. 215; Herv. d. Verf.). Dabei ist wichtig festzuhalten, dass Widerstand bereits im Rahmen der antiken Erörterung als Reaktion auf Unrecht zu verstehen ist und sich gegen zweierlei - hier nur graduell unterschiedene - Arten von Unrecht wenden kann, ohne dass im Anschluss daran verschiedene Formen des Widerstandsrechts systematisch unterschieden würden. Neben dem Widerstand gegen einen Herrscher, der die Gemeinschaft des Rechts aufgekündigt hat, ist auch Widerstand gegen einen tyrannisch agierenden, aber nach wie vor rechtmäßigen Herrscher denkbar. So erörtert Aristoteles auch den Fall, in dem Könige (als rechtmäßige Herrscher) von innen gestürzt werden, nämlich dann, ,wenn (die Könige) versuchen, in einer $z u$ stark tyrannischen Weise zu regieren, (und dies ist der Fall) wenn sie beanspruchen, wider das Recht absolute Macht über eine zu große Zahl von Angelegenheiten zu haben“" (Aristoteles 1996, S. 80; Herv. d. Verf.). 
Mit dem Aufkommen des Vertragsgedankens, zunächst des mittelalterlichen Herrschaftsvertrags, wird legitime Herrschaft und damit Widerstand explizit durch die Rechtsbindung der Herrschenden wie der Beherrschten begründet, wodurch es zugleich zu einer Ausdifferenzierung der in der Antike bereits angelegten Formen des Widerstands kommt; der dort bereits genannte graduelle Unterschied zwischen Tyrann und tyrannisch regierendem König wird nun Grundlage einer systematischen Unterscheidung (vgl. Stüttler 1972). Thomas von Aquin, der die mittelalterlichen Überlegungen zum Widerstand bündelt (vgl. Klautke 1994, S. 192) und erstmals rechtlich präzisiert (Münkler 1995, S. 693), unterscheidet im Sentenzen-Kommentar explizit zwei Formen des Widerstands, je nachdem ob sich dieser gegen eine unrechtmäßige oder eine eigentlich rechtmäßige, aber entartete Herrschaft richtet (Aquin 2019, II, dist. 44, qu. 2 a. 2; vgl. Lewy 1960, S. 67 Anm. 7). Während eine nicht auf göttlichem Recht beruhende und damit illegitime Herrschaft für Aquin ein Fall für den Tyrannenmord, sprich für gewaltsamen Widerstand einzelner, ist (Aquin 2019, II, dist. 44, qu. 2 a. 2, ad 5), ${ }^{10}$ kann gegen einen Herrscher, der göttliche Gebote missachtet oder seine Kompetenz überschreitet, nur systemintern und kollektiv nach ,allgemeinem Beschluss“ (Aquin 2004, S. 24) vorgegangen werden, d.h. vor dem Hintergrund des Herrschaftsvertrags: nach Beschluss der Stände, die politische Autorität ausüben. Bei Thomas wird zugleich die reagierende Qualität der durch das Widerstandsrecht legitimierten Gewalt deutlich, wenn er darauf verweist, dass nicht diejenigen, die gegen den Tyrannen vorgehen, sondern der Tyrann selbst Initiator der Gewalt ist: „Der eigentliche Aufrührer [...] ist der Tyrann“ (Aquin 1933 ff., II/II, qu. 42 , a.2, ad.3).

Die hugenottischen Monarchomachen ${ }^{11}$ beginnen das Widerstandsrecht vor dem Hintergrund der konfessionellen Spaltung säkular zu begründen. Sie waren, weil sich die politische Herrschaft ,mit einer bestimmten, nun zur Konfession werdenden Kirche identifizierte“, gezwungen, ,ihre Position nicht nur theologisch, sondern auch politisch-theoretisch zu begründen“(Dennert 1968, S. XXXIX). Als Höhepunkt des Widerstandsdenkens der Monarchomachen kann Brutus' Vindicae contra tyrannos von 1579 gelten, nicht zuletzt aufgrund einer nun auch weitgehend unabhängigen säkularen Begründung des Widerstandsrechts (vgl. Brutus 1968, S. 129-130). Auch Brutus unterscheidet einen Tyrannen, ,der durch Gewalt oder üble Machenschaften sich der Regierung bemächtigt hat“ und einen, ,der das ihm freiwillig und gern übertragene Reich wider göttliches sowie menschliches Recht regiert und es im Widerspruch zu Gesetzen und Verträgen, auf die er sich hochheilig verpflichtet hat, mit Starrköpfigkeit verwaltet“ (Brutus 1968, S. 165; Herv. d. Verf.). Auch hier differie-

\footnotetext{
10 Die Meinungen in der Forschung gehen hier auseinander: Für Spindelböck (1994) steht fest, dass die Tyrannentötung für Thomas ,nur Ausnahme sein [kann]: falls kein Rekurs bei einer höheren urteilsprechenden Instanz möglich ist“ (S. 87); dagegen, meint J.-B. Klautke (1994), man werde „die Aussage so interpretieren müssen, daß der Antiquate sich die Auffassung Ciceros zu eigen macht“ (S. 199).

11 Die Bezeichnung „Monarchomachen“ („Königsbekämpfer“) geht zurück auf eine von William Barclay 1600 publizierte Polemik, die sich gegen eine Gruppe calvinistischer Publizisten wendet, die auch als Reaktion auf die Bartholomäusnacht (1572) die uneingeschränkte Herrschaftsgewalt der Monarchen infrage stellten. Ihrem Selbstverständnis nach kämpften die Monarchomachen nicht gegen den König, sondern gegen den Tyrannen. Die bekanntesten Vertreter sind die Franzosen François Hotman, Theodor Beza und der Anonymus Brutus.
} 
ren in Abhängigkeit von der Ursache bzw. dem Verursacher Akteure und Mittel des Widerstands. Im Falle einer illegitim errichteten Gewaltherrschaft ist es laut Brutus ,jedem beliebigen Privatmann erlaubt, sie abzuwehren“ - kein Eid, kein Vertrag, keine öffentliche oder private Verpflichtung steht der Gewaltanwendung in diesem Fall im Wege (Brutus 1968, S. 173). Das Widerstandsrecht gegen einen ursprünglich legitimen Herrscher liegt dagegen allein bei den Ständen, die ,die Rechte und Vorrechte des Volkes schützen und genau darauf achten, daß der Herrscher nichts zum Verderben des Volkes begeht oder unterläßt“ (Brutus 1968, S. 118), hier soll man zunächst ,,jedes Mittel eher versuchen, als die Waffen“; hört der Herrscher nicht auf die Warnungen, dann ,kann man alles gegen ihn unternehmen, was das Recht und die berechtigte Notwehr gegen einen Tyrannen erlauben“ (Brutus 1968, S. 177). Dann sind die Amtspersonen verpflichtet, den Tyrannen entsprechend den Gesetzen zu richten und falls er sich widersetzt, gewaltsam gegen ihn vorzugehen: dann kommt es den Repräsentanten des Volkes zu, ,das Volk zu den Waffen zu rufen, ein Heer aufzustellen und gegen ihn wie gegen einen Feind des Vaterlandes und des Gemeinwesens Gewalt, List und jedes andere Mittel anzuwenden“ (Brutus 1968, S. 178). Auch hier ist die Gewalt nicht initiativ, sie ist eine Reaktion auf vorangegangenes Unrecht - nicht die, ,welche gegen ihn die Waffen erheben“, können „mit dem Verbrechen der Majestätsverletzung belastet werden“, es ist „,der Gewaltherrscher selbst, der sich des genannten Verbrechens schuldig macht“ (Brutus 1968, S. 179).

Trotz der säkularen Anklänge bleiben Herrschaft und Widerstand, wie zuvor bei Aquin, auch bei den Monarchomachen in letzter Konsequenz religiös begründet und hinsichtlich der Frage nach ihrer Legitimation auf eine dazu berufene, in dieser Hinsicht privilegierte Minderheit verwiesen. Anders gesagt: Der Herrschaftsvertrag regelt zwar die gleiche Bindung an höheres, göttliches Recht, die Auslegung dieses Rechts aber ist den Herrschenden vorbehalten. Mehr noch setzt der Herrschaftsvertrag ,eine fertige soziale und politische Ordnung voraus, von der nicht angenommen wird, daß sie durch Vertrag entstanden sei oder entstehen könnte: eine ständisch gegliederte Gesellschaft, deren Mitglieder auf unterschiedliche Weise über Rechte und Privilegien verfügen“" (Ballestrem 1983, S. 2). Der mittelalterliche Herrschaftsvertrag rechtfertigt folglich die gleiche Bindung der Herrschenden und Beherrschten an ungleiches Recht. Der institutionalisierte Schutz vor herrschaftlicher Willkür ebenso wie das Recht auf Widerstand - das sich durch die Befreiung von Willkürherrschaft rechtfertigt, wo die institutionalisierten Vorkehrungen versagen oder nicht vorhanden sind - müssen so im Rahmen des mittelalterlichen Herrschaftsvertrags unvollständig bleiben. Seine moderne, individualistische und damit demokratische Gestalt, die das Ziel der Befreiung von Willkürherrschaft konsequent ausbuchstabiert, erhält das Widerstandsrecht erst im Rahmen der neuzeitlichen Theorie des Gesellschaftsvertrags.

Den Höhepunkt der Ausdifferenzierung und Säkularisierung des Widerstandsrechts bildet das Denken John Lockes, der das Widerstandsrecht zugleich demokratisiert. Erst hier wird auch die Auslegung des höheren Rechts, sprich die Setzung positiven Rechts, selbst vor herrschaftlicher Willkür geschützt, indem sie demokratisch und machtteilig organisiert wird. Der von Locke konzipierte Vertrag ist ein Gesellschafts-, kein Herrschaftsvertrag. Der Vertrag konstituiert die Gesellschaft, 
die Herrschaft dagegen ist nur treuhändisch verliehen. Lockes Regierung beruht auf „trust“ (vgl. Dunn 1984). Die zur Gesellschaft vereinten Individuen vertrauen ihre Macht der Legislative, deren Einsetzung dem Vertragsschluss unmittelbar folgt und die entsprechend die höchste Gewalt ist, und vermittels dieser der Exekutive an, auf dass beide sie zum Wohle der Gemeinschaft einsetzen. Der Widerstandsfall tritt für Locke in zwei Fällen ein: „First, When the Legislative is altered“ (Locke 1988, $\S 212$; Herv. i. O.) und ,secondly [...] when the Legislative, or the Prince, either of them act contrary to their Trust" (Locke 1988, § 221). Wie Nathan Tarcov (1999) überzeugend zeigt, hat beides völlig unterschiedliche Konsequenzen: Während die Änderung der Legislative die Auflösung der Gesellschaft (sprich einen Rückfall in den Zustand vor Vertragsschluss) nach sich zieht, führt ein Vertrauensbruch durch die Träger staatlicher Gewalt nur zur Absetzung der Regierung. Dem entsprechen zwei Fälle des Widerstandsrechts: Während im ersten Fall - wie auch im Fall von Eroberung, Usurpation und Tyrannei - jedem Individuum ein Widerstandsrecht zukommt, liegt das Widerstandsrecht im Falle eines Vertrauensbruchs bei der Mehrheit der zur Gesellschaft vereinten Individuen. Die Gesellschaft besteht in diesem Fall fort, das kollektive systeminterne Widerstandsrecht soll die Tyrannei verhindern, wird dagegen die Legislative geändert, bedeutet dies die Errichtung einer Willkürherrschaft (,the exercise of Power beyond Right" [Locke 1988, § 199; Herv. i. O.]) und führt zur Auflösung der Gesellschaft. Denn die Legislative

is the Soul that gives Form, Life, and Unity to the Commonwealth: From hence the several Members have their mutual Influence, Sympathy, and Connexion: And therefore when the Legislative is broken, or dissolved, Dissolution and Death follows. For the Essence and Union of the Society consisting in having one Will, the Legislative (Locke 1988, § 212; Herv. i. O.).

Wird die Legislative geändert - das heißt bemächtigen sich Unbefugte ihrer unrechtmäßig, zerstören oder marginalisieren sie -, gibt es keinen einheitlichen Willen mehr (Locke 1988, § 212), es herrscht Willkür, die Gesellschaft ist in diesem Fall aufgelöst, individueller Widerstand ist erlaubt. Auch Locke versteht widerständische Gewalt dabei stets als Gegengewalt: „For when Men by entering into Society and Civil Government, have excluded force [...]; those who set up force again in opposition to the Laws, do Rebellare, that is, bring back again the state of War, and are properly Rebels“ (Locke 1988, § 226).

Entscheidend für die vorliegende Untersuchung ist nun, dass die klassische Theorie des Widerstandsrechts einen Maßstab für die normative Legitimation politischer Gewalt enthält, der von der inhaltlichen Bestimmung des jeweils adressierten höheren Rechts unabhängig ist, sprich von moralischen Überzeugungen abstrahiert: die Befreiung von Willkürherrschaft durch die Rechtsbindung der Herrschenden und Beherrschten. Nimmt man die Forderung nach der Befreiung von Willkürherrschaft ernst, muss dies auch die Rechtssetzung selbst, sprich die Auslegung des höheren Rechts, einschließen. Erst die Theorie des Gesellschaftsvertrags denkt den Schutz vor herrschaftlicher Willkür und damit die Rechtfertigung von Widerstand gegen Willkürherrschaft konsequent zu Ende: Um Willkürherrschaft zu vermeiden, müssen Herrschende und Beherrschte gleichermaßen an gleiches Recht gebunden sein. Insofern politische Gewaltakteure ihrem Selbstverständnis nach Widerstand leisten, 
d.h. unter Berufung auf höheres Recht auf die Beseitigung einer willkürlichen Herrschaft zielen und diese durch eine Herrschaft ersetzen wollen, in der Herrschende und Beherrschte gleichermaßen dem höheren Recht unterworfen sind, lassen sie sich daran messen. Der in der Rechtfertigung illegaler politischer Gewalt als Widerstand gegen Willkürherrschaft enthaltene Maßstab kann so zur Kennzeichnung einer ihrem Selbstverständnis nach auf die Befreiung von Willkürherrschaft zielenden politischen Gewalt als legitim bzw. illegitim dienen. Legitim ist illegale politische Gewalt demzufolge zunächst dann, wenn sie sich gegen eine Herrschaft richtet, in der Herrscher und Beherrschte nicht gleichermaßen gleichem Recht unterworfen sind und das heißt gegen eine Herrschaft, die nicht demokratisch und machtteilig organisiert ist.

Zugleich werden durch die systematische Ausdifferenzierung zweier Rechte anhand der Qualität des konstatierten Unrechts, auf das sie reagieren, zwei unterschiedliche Zielsetzungen des Widerstands expliziert. Die klassische Theorie des Widerstandsrechts, der es vor allem um die Rechtfertigung eines systeminternen, konservierenden Widerstands geht, endet gewissermaßen in der Neuzeit. Lockes Überlegungen zum kollektiven Widerstandrecht werden im modernen Rechtsstaat vom Verfassungsrecht und der Verfassungsgerichtsbarkeit absorbiert; es wird mit dem Aufkommen des modernen Verfassungsstaates obsolet: „Mit dem Ausbau des Rechtsstaats auf konstitutioneller Grundlage war daher die Widerstandslehre soziologisch erledigt. Ihr rechtlich-sittlicher Grundgedanke war erfüllt, ihre logische Daseinsberechtigung aufgelöst" (Wolzendorff 1968, S. 534). Das gilt gleichwohl nur für das systeminterne kollektive Widerstandsrecht als notwendige Ergänzung eines unvollständig entwickelten Gewaltenteilungssystems (vgl. Höntzsch 2010), nicht aber für das Konzept des zivilen Ungehorsam, das sich als Residuum des kollektiven Widerstandsrechts verstehen lässt (sprich als der Teil des kollektiven Widerstandsrechts, der nicht in Verfassungsrecht überführbar ist und folglich im demokratischen Rechtsstaat erhalten bleibt), und auch nicht für das Widerstandsrecht gegen Willkürherrschaft. ${ }^{12}$ Während ziviler Ungehorsam (als Nachfolger des kollektiven Widerstandsrechts) das System als Ganzes nicht infrage stellt (und Gewalt gegen Personen als Mitbürger notwendig ausschließt [vgl. Celikates 2010, S. 294]), zielt Widerstand gegen Willkürherrschaft auf die notfalls gewaltsame Beseitigung einer als illegitim erachteten Herrschaft. Widerstand ist in seiner theoretischen Anlage folglich keineswegs notwendig konservierend, wie das normalerweise konstatiert wird, ${ }^{13}$ ganz im Gegenteil: Widerstand gegen Willkürherrschaft besitzt mittelbar stets revolutionären Charakter, insofern er mit der Beseitigung eines Unrechtsregimes mit-

\footnotetext{
12 Die theoretische Beschäftigung mit Widerstand konzentriert sich allerdings im Anschluss überwiegend auf das Konzept des zivilen Ungehorsams (vgl. Thoreau 1966; Rawls 1979, S. 399-430; Habermas 1983; Arendt 2000; Markovits 2005; Celikates 2010).

13 Vgl. exemplarisch den Beitrag „Widerstandslehren“ im Lexikon der Politik von Herfried Münkler (1995), der zwar auf die revolutionäre Verwendung des Begriffs im Rahmen der französischen ,Erklärung der Menschen- und Bürgerrechte“ von 1789 hinweist, aber gerade in Abgrenzung dazu festhält: „Eine präzisierende Rekonstruktion des Begriffs Widerstand muß diesen also gegen revolutionäre Handlungen abgrenzen und auf die Verteidigung des Status quo bzw. die Wiederherstellung des Status quo ante festlegen" (S. 692).
} 
telbar zugleich auf eine Neuordnung zielt. ${ }^{14}$ In Lockes Worten ist das Individuum in diesem Falle wieder frei ,to shift for himself, and provide for his own Safety as he thinks fit in some other Society“ (Locke 1988, § 211; Herv. d. Verf.). Widerstand gegen bzw. als Befreiung von Willkürherrschaft ist so, über das mittelbare Ziel der Neuordnung, mit dem neuzeitlichen Revolutionsbegriff verbunden.

\section{Revolution als mittelbares Ziel von Widerstand $u$ nd Terrorismus}

\subsection{Widerstand als (aller)erste Phase der Revolution}

Abhandlungen über Revolution beginnen oftmals mit dem Hinweis darauf, dass die Verwendung des Begriffs im modernen Wortsinn auf die Französische Revolution zurückgehe, während Revolution bis dahin im Gegensatz dazu gerade ,die Rückkehr zu einem früheren Zustand“ (vgl. Enzmann 2013b, S. 206) bzw. ,ein Zurückschwingen zu einer zuvor vorhandenen Ordnung“ (Arendt 2012, S. 236) bezeichnet habe. Naheliegender als die Annahme einer Wandlung oder gar Verkehrung des Begriffs scheint mir jedoch die Annahme, dass sich hier eine Ausdifferenzierung parallel zur Unterscheidung der zwei Formen des Widerstands vollzieht. Dafür spricht, dass Widerstand und Revolution in klassischen Erörterungen in einem engen begrifflichen Zusammenhang stehen und teilweise synonym verwandt werden. ${ }^{15}$ So auch bei Locke (vgl. 1988, §§ 224-225), in dessen Verwendung des Begriffs der Revolution, parallel zur Unterscheidung zweier Formen des Widerstands, eine konservierende wie eine systemumstürzende Bedeutung aufscheint, ${ }^{16}$ ohne dass die verschiedenen Bedeutungen hier differenziert würden. Mit Blick auf den möglichen Vorwurf, ein Widerstandsrecht führe zu ständiger Rebellion, stellt er, die Beharrungskräfte des Volkes betonend, fest: „This slowness and aversion in the People to quit their old Constitutions, has, in the many Revolutions which have been seen in this Kingdom [...] still kept us to, or, after some interval of fruitless attempts, still brought

\footnotetext{
14 Der Sache nach ähnlich unterscheidet Christopher Daase (2014, S. 3) begrifflich zwischen Opposition und Dissidenz, wenn er auch dem Widerstand eine konservative Absicht zuschreibt und ihn ausdrücklich von der Revolution abgrenzt: ,[D]em Widerstand [liegt], anders als der Revolution, zumindest deklaratorisch eine konservative Absicht zugrunde. Ausgeschlossen ist allerdings nicht, dass sich eine Widerstandsbewegung zu revolutionären Zielen bekennt, wenn sie damit die Wiederherstellung eines legitimen „Urzustands" beabsichtigt. Widerstand kann somit systemimmanent, also im Rahmen der Spielregeln eines politischen Systems, ausgeübt werden oder sich unkonventioneller Mittel bedienen und auf Systemüberwindung zielen." Ersteres bezeichnet Daase als Opposition, Letzteres als Dissidenz.

15 Das zeigt sich sogar noch bei Henry David Thoreau, der als Namensgeber des zivilen Ungehorsams gilt: „All men recognize the right of revolution; that is, the right to refuse allegiance to and to resist the government, when its tyranny or its inefficiency are great and unendurable“ (Thoreau 1966, S. 227).

16 Locke grenzt in diesem Zusammenhang das kollektive Widerstandsrecht/die konservierende Revolution als legitim begrifflich von „Rebellion“ als im konstitutionellen Staat illegitime, weil gewaltsame Form des Widerstands ab: „For Rebellion being an Opposition, not to Persons, but Authority, which is founded only in the Constitutions and Laws of the Government; those, whoever they be, who by force break through, and by force justifie their violation of them, are truly and properly Rebels" (Locke 1988, § 226; Herv. i. O.). Ähnlich hält Arendt mit Blick auf den zivilen Ungehorsam fest: ,,Von allen Mitteln, derer sich die Akteure zivilen Ungehorsams bei der Überzeugung anderer und bei der Darstellung ihrer Sache bedienen mögen, rechtfertigt nur eines ihre Bezeichnung als ,Rebellen“: das Mittel der Gewalt“ (Arendt 2000, S. 301).
} 
us back again to our old Legislative of King, Lords and Commons" (Locke 1988, $\S 223$; Herv. d. Verf.). Während der konservierende Revolutionsbegriff, der sich auf den Erhalt der bestehenden Ordnung bezieht, wie das kollektive Widerstandsrecht im modernen Verfassungsstaat obsolet zu werden scheint, ist mit dem modernen Begriff der Revolution, der sich im Anschluss an die Französische Revolution durchsetzt, Widerstand gegen Willkürherrschaft unauflöslich verknüpft. Widerstand gegen Willkürherrschaft richtet sich gegen das bestehende als unrechtmäßig erachtete System und zielt folglich mittelbar auf einen Systemwechsel.

Deutlich wird dieser Zusammenhang zwischen Widerstand (gegen Willkürherrschaft) und Revolution (im modernen Sinne) der Sache nach bei Hannah Arendt, gleichwohl sie selbst in diesem Zusammenhang nicht von „Widerstand“, sondern von „Befreiung“ spricht. Arendt geht davon aus, „daß jede Revolution zwei Stadien durchzumachen hat: das Stadium der Befreiung - von Armut und Fremdherrschaft und das Stadium der Gründung der Freiheit. Unter dem Gesichtspunkt eines politischen Prozesses gehören die beiden zusammen, und doch sind sie als politische Phänomene vollkommen verschieden und müssen auseinandergehalten werden" (Arendt 2012, S. 249). Arendt geht es in erster Linie um die Gründung der Freiheit, wobei sie den Umschlagspunkt zwischen Befreiung und dieser in ihren Augen eigentlichen Revolution (der Gründung der Freiheit) nicht zuletzt an der Frage der Gewalt festmacht. Mit Blick auf die Französische Revolution hält sie fest: „Die erste Entwicklungsetappe war von Gewalt gekennzeichnet; gegen die Gewalt der Tyrannei, die Verletzung menschlicher Rechte und Möglichkeiten, mußte die Gewalt der Befreiung gesetzt werden. Von der zweiten Etappe nahm man an, daß sie frei von Gewalt sein werde“ (Arendt 2012, S. 240).

In der Revolutionsforschung wird Gewalt erst in neuerer Zeit nicht mehr als notwendiges Merkmal der Revolution angesehen; zwar wird nach wie vor von einem potenziell gewaltsamen Charakter ausgegangen, aber ohne dass es tatsächlich zur Gewaltanwendung kommen muss (vgl. u.a. Colgan 2012). Drei Merkmale werden in den meisten Definitionen angeführt: als Ziel die Umwälzung bzw. Veränderung der vorgefundenen (politischen, gesellschaftlichen, ökonomischen) Ordnung, als $A k$ teure breite Bevölkerungsschichten und als Mittel nichtinstitutionalisierte Aktionen (die auch Gewalt einschließen können, aber nicht müssen). Dieter Wolf und Michael Zürn etwa definieren Revolutionen als ,tiefgreifende und nachhaltige Veränderungen der Struktur eines politischen (und häufig auch gesellschaftlichen) Systems [...], die vergleichsweise abrupt erfolgen und sich auf der Grundlage einer breiten Bevölkerungskoalition vollziehen“ (Wolf und Zürn 1995, S. 552). Jack Goldstone fasst Revolution als ,,an effort to transform the political institutions and the justifications for political authority in a society, accompanied by formal or informal mass mobilization and noninstitutionalized actions that undermine existing authorities“" (Goldstone 2001, S. 142).

Dabei scheint mir die Frage nach den Akteuren für die Abgrenzung zum Widerstand zentral, die Frage nach dem potenziellen Einsatz von Gewalt dagegen nur für die Unterscheidung zwischen der Phase der Befreiung und des Prozesses der Neuordnung: So zielt Widerstand gegen Willkürherrschaft, wie Revolution, auf eine vergleichsweise abrupte Umwälzung bzw. Veränderung der vorgefundenen (politischen, gesellschaftlichen, ökonomischen) Ordnung und bedient sich als Mittel 
nichtinstitutionalisierter Aktionen (die auch Gewalt einschließen können, aber nicht müssen, auch Widerstand gegen Willkürherrschaft ist nur potenziell gewaltsam), sodass sich als einziger Unterschied die tragenden Akteuren ausmachen lassen. Das heißt: Lässt sich die erste Phase der Revolution bzw. der Beginn jeder Revolution auch mit Arendt als Stadium der Befreiung verstehen, muss dieses doch noch einmal in sich differenziert werden: So steht am Beginn der Befreiung von Unterdrückung und Willkür der Widerstand (einer Minderheit), der im Falle einer gelingenden Massenmobilisierung vom Begriff der „Revolution“ gewissermaßen absorbiert wird; Befreiung gliedert sich also in einen durch eine Minderheit initiierten und von den Massen zu vollziehenden Umsturz. Implizit findet sich das auch bei Arendt, wenn sie zwischen den „Männern der Revolution“ und „,er Menge in den Straßen“ unterscheidet (Arendt 2012, S. 242).

Insofern Widerstand als erste Phase der Revolution (bzw. genauer: als erste Phase der Befreiung als erste Phase der Revolution) zu verstehen ist, und also zwar zunächst auf die Beseitigung der bestehenden Ordnung, mittelbar aber auf eine Neuordnung zielt, lässt sich der Maßstab für die Legitimation politischer Gewalt anhand ihrer unmittelbaren und mittelbaren Zielsetzung ausdifferenzieren: Während die Rechtmäßigkeit politischer Gewalt mit Blick auf die Befreiung von Willkürherrschaft zunächst daran zu messen ist, ob die angegriffene Ordnung die Bindung der Herrschenden und Beherrschten an gleiches Recht missachtet, misst sich ihre Rechtmäßigkeit in einem weiteren Schritt (in der Phase der auf den Umsturz folgenden Neuordnung bzw. anhand der von den Gewaltakteuren formulierten Ziele hinsichtlich der Neuordnung) daran, ob sich das Anliegen - die Befreiung von Willkürherrschaft auch in der Organisation des Prozesses der Neuordnung widerspiegelt, ob also der Prozess der Neuordnung demokratisch und machtteilig organisiert wird bzw. werden soll. Das heißt: Die Rechtfertigung des Widerstands gegen Willkürherrschaft impliziert ex negativo einen demokratisch und machtteilig organisierten Prozess der Neuordnung. Vor diesem Hintergrund ist plausibel, dass man mit Arendt nur im Falle einer Gründung in Freiheit von einer erfolgreichen Revolution sprechen kann, also dann, wenn der demokratisch und machtteilig organisierte Prozess der Neuordnung in eine demokratische und machtteilige neue Ordnung mündet, sodass sich die Rechtmäßigkeit politischer Gewalt zuletzt auch anhand der angestrebten Ordnung messen lässt. Auch die Gründung der Freiheit, als zweite Phase der bzw. mit Arendt als eigentliche Revolution, lässt sich folglich in sich noch einmal differenzieren: in den Prozess der Neuordnung und die Konstitution der neuen Ordnung.

\subsection{Terrorismus $=$ revolutionärer Terrorismus}

Wie das Konzept des gewaltsamen Widerstands ist auch das Konzept des Terrorismus in seinen theoretischen Ursprüngen mit der Revolution verbunden; sei es im Anschluss an die Französische Revolution, die in ,terreur“ umschlägt, sei es im Rahmen des russischen Anarchismus, der den Terror im Sinne der Propaganda der Tat als Mittel der Revolutionäre versteht; in diesem Umfeld finden sich jedenfalls die vergleichsweise spärlichen ideengeschichtlichen Quellen zum Konzept des ,Terrorismus“, die gleichwohl, so sie von „Terrorismus“ sprechen, diesen nicht (auch wenn sie sich zum „Terror“ bekennen) als Selbstbezeichnung wählen, sondern als antizi- 
pierte Fremdzuschreibung. Man kann so insbesondere die anarchistischen Texte als Texte einer nichtliberalen Widerstandstradition lesen, was oftmals nicht geschieht, weil sie stärker auf die Revolution (als mittelbares Ziel des Widerstands) fokussieren als auf die Rechtfertigung der dazu erforderlichen Gewalt. ${ }^{17}$

Sind auch die Ausführungen Maximilien de Robespierres im vorliegenden $\mathrm{Zu}-$ sammenhang weniger interessant, weil es sich beim ,terreur" der Jakobiner letztlich um eine Spielart des Staatsterrors handelt, verweist doch der Umstand, dass der "terreur" aus der Revolution hervorging, auf den inhärenten Zusammenhang der Konzepte des gewaltsamen Widerstands, der Revolution und des Terrorismus. Arendt hält mit Blick auf die Französische Revolution fest: „Der Terror und nicht so sehr die bloße Gewalt - der Terror, der losgelassen wird, nachdem das alte Regime besiegt und das neue errichtet ist - stürzt die Revolutionen ins Verderben" (2012, S. 245). Die Französische Revolution war laut Arendt in dem Moment gescheitert, als das „Glück des Volkes“ zum Ziel der Revolution erklärt wurde: „Das erste Anzeichen des Untergangs ist da, wenn diejenigen, die neu an die Macht gekommen sind, zu vergessen beginnen, daß Ziel und Ende der Revolution einzig die Freiheit ist“" (Arendt 2012, S. 245). In diesem Verständnis delegitimiert sich - und das entspricht der vorgeschlagenen Konzeptualisierung - politische Gewalt, wo das Ziel der Befreiung von Willkürherrschaft nicht in die politische Neuordnung übersetzt wird, sprich wo bereits der Prozess der Neuordnung nicht demokratisch und machtteilig organisiert wird oder wie im Falle der Terrorherrschaft der Jakobiner keine machtteilige, demokratische Ordnung installiert wird, die Herrscher und Beherrschte gleichermaßen an gleiches Recht bindet, sondern stattdessen die Ziele einer Minderheit (die als „Wohl des Volkes“ deklariert werden) absolut gesetzt und gewaltsam durchgesetzt werden. So hielt Robespierre in seiner Rede vor dem Konvent vom 25. Dezember 1793 ,Über die Grundsätze der revolutionären Regierung“ den „,erreur“ begründend fest: „Wenn die revolutionäre Regierung in ihrer Arbeit aktiver und freier sein muß als die gewöhnliche Regierung, ist sie deshalb weniger gerecht und weniger legitim? Nein, sie stützt sich auf das heiligste aller Gesetze, nämlich auf das Wohl des Volkes, und auf die unbestreitbarste aller Vollmachten, nämlich auf die Notwendigkeit“ (Robespierre 1989, S. 566).

Eine mit Blick auf nichtstaatlichen Terrorismus wichtigere, ideengeschichtliche Quelle für das Konzept des Terrorismus bilden die Schriften der russischen Anarchisten, vor allem diejenigen von Michail Bakunin. Auch Bakunin unterscheidet zwei Phasen der Revolution: „In Bezug auf die Zeit enthält der Begriff Revolution zwei gänzlich verschiedene Tatsachen: den Anfang, die Zeit der Zerstörung der vorhandenen sozialen Formen, und das Ende, den Aufbau, d.h. die Bildung vollkommen neuer Formen aus diesem Amorphismus“ (1972, S. 101-102; Herv. i. O.). Die erste Phase erlaubt auch Gewalt: „Wenn wir auch keine andre Tätigkeit als die Sache der Zerstörung anerkennen, so sind wir dennoch der Meinung, daß die Formen, in denen diese Tätigkeit sich äußern mag, außerordentlich mannigfaltig sein können. Gift, Dolch, Schlinge u. dergl.!... Die Revolution heiligt alles in diesem

\footnotetext{
17 Mit Blick auf transnationale Formen gewaltlosen Widerstands dagegen finden sich neben Ansätzen, die in der liberal-konstitutionalistischen Tradition stehen, auch solche die auf die postmarxistisch-anarchistische Tradition rekurrieren; vgl. dazu Celikates und Höntzsch (2019).
} 
Kampfe in gleicher Weise. Das Feld ist also frei!““ (Bakunin 1972, S. 104) und Bakunin prophezeit, die Fremdzuschreibung antizipierend: „Man wird es Terrorismus nennen“ (1972, S. 104). Auf dieses anarchistische Verständnis rekurriert auch J. B. S. Hardman zur Bestimmung des Begriffs „Terrorismus“ in der Encyclopedia of Social Sciences: ,,(T)errorism in its proper sense implies open defiance of law and is the means whereby an opposition aims to demoralize a governmental authority, to undermine its power and to initiate a revolution or counter-revolution" (1948, S. 576). Terrorismus wird hier als revolutionäre Taktik einer Minderheit verstanden, die darauf zielt, eine Revolution zu initiieren. Dabei wird betont, so Hardman, ,that the use of terror was not an end in itself but only a means of effect the transference of power from the government to the people" (Hardman 1948, S. 578). Der Terror wird hier folglich als ein Mittel der Ermächtigung, der Übertragung der Macht auf das Volk verstanden. Die Parallelen dessen, was hier als Terrorismus gefasst wird, zum Begriff des Widerstands gegen Willkürherrschaft als der ersten, gewaltsamen Phase der Revolution, die den Umsturz initiiert und über diesen hinaus auf eine demokratisch organisierte Neuordnung zielt, ist nicht zu übersehen; von Terroristen in Abgrenzung zu Widerständlern lässt sich auf dieser Grundlage jedenfalls nicht sinnvoll sprechen.

In der Terrorismus-Forschung wird der revolutionäre Terrorismus (vgl. Crenshaw 1972) gleichwohl als nur eine Spielart neben dem ethnisch-nationalistischen Terrorismus und dem religiösen/fundamentalistischen/islamistischen Terrorismus (teils ergänzt durch einen rechtsradikalen Terrorismus) genannt (vgl. Waldmann 2005, S. 99-100), wobei unter revolutionärem Terrorismus zumeist ein sozialrevolutionärer Terrorismus in Weiterentwicklung eines orthodoxen Marxismus gefasst wird, der wie der ethnisch-nationalistische mit dem ,,alten Terrorismus“ gleichgesetzt wird (vgl. Waldmann 2005, S. 17; Schrader 2002); das heißt das Ziel des Regimewechsels wird in diesem Verständnis mit einer bestimmten Weltanschauung verbunden, revolutionärer Terrorismus als sozialrevolutionär über die moralischen Überzeugungen der Akteure definiert. Auch die zeitliche Unterteilung der genannten Spielarten des modernen Terrorismus in vier aufeinanderfolgenden Wellen durch David Rapoport (2004) - die anarchistische Welle, die antikoloniale Welle, die „Neue Linke“-Welle und die religiöse Welle (wobei hier der Anarchismus und die Neue Linke dem revolutionären Terrorismus zuzuordnen wären) - spricht dafür, dass es sich bei den verschiedenen Spielarten primär um eine Unterscheidung anhand der je zugrundeliegenden moralischen Überzeugungen handelt. Konzipiert man Terrorismus dagegen, wie hier vorgeschlagen, über die ihm in seiner Eigenschaft als illegale politische Gewalt inhärente Zielsetzung der Beseitigung einer willkürlichen Herrschaft und der darin impliziten mittelbaren Zielsetzung der Neuordnung, dann ist der ,revolutionäre Terrorismus“ nicht einfach eine Spielart des Terrorismus, sondern ist die als Terrorismus bezeichnete politische Gewalt in ihrer Zielsetzung mittelbar stets revolutionär. ${ }^{18}$

\footnotetext{
18 Auch die Unterscheidung zwischen altem und neuem Terrorismus (erstmals bei Laqueur 1996; vgl. auch Laqueur 1999; Neumann 2009; vgl. dagegen u. a. Daase 2013, S. 339) ist mit Blick auf das Ziel der Befreiung von einer als willkürlich erachteten Herrschaft bzw. des Umsturzes des bestehenden Systems, kaum aufrechtzuerhalten; wenn auch im Falle des sogenannten ,,islamistischen Terrorismus“ die Veränderung der globalen Ordnung als Vorbedingung für den Systemwechsel im Laufe der Zeit miteinbezogen wurde (vgl.
} 
Diese Sichtweise wird bestätigt durch Andrew Kydds und Barbara Walters Analyse der Ziele terroristischer Organisationen: ,Although the ultimate goals of terrorists have varied over time, five have had enduring importance: regime change, territorial change, policy change, social control, and status quo maintenance" (Kydd und Walter 2006, S. 52). Dabei werden die im Folgenden von Kydd und Walter tabellarisch angeführten 42 terroristischen Vereinigungen sämtlich (bis auf eine Gruppe, als deren Ziel ausschließlich ,status quo maintenance“ angegeben ist) auch unter „regime change“ (31) und/oder ,territorial change“ (19) gefasst (Kydd und Walter 2006, S. 54), wobei ,territorial change“ als ,taking territory away from a state either to establish a new state [...] or to join another state“" (Kydd und Walter 2006, S. 52) in den meisten Fällen mit einem Regimewechsel einhergehen dürfte.

Vor dem Hintergrund der Parallelität der angestrebten Ziele (Befreiung von Willkürherrschaft und damit mittelbar Neuordnung), der handelnden Akteure (Minderheit) und der verwendeten Mittel ([auch] Gewalt) wird deutlich, dass die Differenz zwischen Terrorismus und gewaltsamem Widerstand als Formen illegaler politischer Gewalt zunächst in der Frage nach der Legitimation liegt und nur abgeleitet, infolge ihrer (De-)Legitimation, auch in der Vorgehensweise bzw. den Kennzeichen der verwendeten Gewalt. Insofern politische Gewaltakteure notwendigerweise von der Legitimation (durch höheres Recht) der von ihnen verübten illegalen politischen Gewalt (im Selbstverständnis: von Gewalt gegen eine nicht an diesem höheren Recht orientierte und deshalb willkürliche Herrschaft) ausgehen müssen, sprich sie sich selbst als Widerständler verstehen müssen, lassen sie sich an der klassischen Rechtfertigung gewaltsamen Widerstands messen. Der Maßstab für die Unterscheidung legitimer und illegitimer Gewalt lässt sich dabei, wie das im Rahmen der Erörterung der Rechtfertigung von gewaltsamem Widerstand durch die Befreiung von Willkürherrschaft als erster Phase der Revolution herausgearbeitet wurde und nun noch einmal zusammenfassend dargelegt werden soll, analytisch in zwei (bzw. drei) Schritte zerlegen (wobei der zweite und dritte Schritt unmittelbar zusammenhängen): Die Rechtfertigung des unmittelbaren Ziels der Beseitigung des bestehenden Systems und des mittelbaren Ziels der Neuordnung durch die Kennzeichnung des bestehenden Systems als Willkürherrschaft (de-)legitimiert politische Gewalt in normativer Hinsicht (unabhängig von der jeweiligen Quelle des Rechts) in einem ersten Schritt dann, wenn sie sich (nicht) gegen eine Herrschaft richtet, die Herrschende und Beherrschte gleichermaßen gleichem Recht unterwirft. In einem zweiten (und dritten) Schritt kann politische Gewalt als legitim bzw. illegitim gekennzeichnet werden, wenn zweitens der Prozess und drittens die Gestalt der Neuordnung dem im Widerstand gegen Willkürherrschaft ex negativo enthaltenen Ziel ent- bzw. widersprechen. Die Rechtfertigung illegaler politischer Gewalt durch die Befreiung von Willkürherrschaft impliziert eine demokratische und machtteilige - als einzig dem Ziel der Befreiung von Willkürherrschaft und damit der Bindung der Herrschenden

dazu etwa Zawahiri; zitiert nach Steinberg 2005, S. 61: „Der Kampf für die Errichtung eines islamischen Staates kann nicht als regionaler Kampf geführt werden. [...] Um uns dieser neuen Realität anzupassen, müssen wir uns für eine Schlacht vorbereiten, die sich nicht auf eine einzelne Region beschränken wird, sondern auf eine, die sowohl den nahen Feind als auch den fernen Feind, die Allianz der Juden und Kreuzzügler einschließt“; vgl. zur Unterscheidung von nahem und fernem Feind Steinberg 2005, S. 10/1 und insbesondere Gerges 2005). 
und Beherrschten an gleiches Recht entsprechende - Organisation des Prozesses der Neuordnung und in letzter Konsequenz (und auch das ist unabhängig von der jeweiligen Quelle des Rechts) auch der neu zu installierenden Ordnung.

\section{Entgrenzung politischer Gewalt als empirischer Marker fehlender Legitimation}

Durch die vorgeschlagene Konzeptualisierung von Terrorismus soll den gängigen Terror(ismus)definitionen nicht ihre Berechtigung abgesprochen werden - ganz im Gegenteil: Indem die in der Terrorismusforschung im Vordergrund stehende Analyse der spezifischen Vorgehensweise an die illegaler politischer Gewalt inhärenten Ziele und damit an die Frage nach der Legitimation rückgekoppelt wird, zeigt sich, dass die Besonderheiten terroristischer Gewalt bzw. die im Rahmen der zahlreichen Terrorismusdefinitionen aufgezählten Merkmale terroristischer Gewalt als Folge ihrer Delegitimation verstanden werden können und sich als Entgrenzung hinsichtlich Zeit, Raum, Qualität, Opfern und Zielen (zusammen)fassen lassen.

Genaugenommen scheint die Entgrenzung politischer Gewalt Folge der ausbleibenden empirischen Legitimation zu sein, die auf die fehlende normative Legitimation verweist: Illegale, politische Gewalt bedarf empirisch, sofern sie von einer Minderheit initiiert wird und nicht über überlegene Gewaltmittel verfügt, um ihr Ziel der Beseitigung der bestehenden Ordnung politisch umzusetzen, der Zustimmung durch die adressierte Öffentlichkeit. Sie kann folglich empirisch nur nachträglich als legitime politische Gewalt gekennzeichnet werden, und zwar innersystemisch nur dann, wenn die Mehrheit der Rechtfertigung der Gewalt durch die jeweiligen Akteure folgt. Wo politische Gewalt sich nicht dem Maßstab der gleichen Bindung der Herrschenden und Beherrschten an gleiches Recht verpflichtet, wird sie - so lässt sich vermuten - keine Mehrheit erlangen; sei es weil bereits die Einschätzung der Unrechtmäßigkeit der bestehenden Ordnung nicht geteilt wird, weil sie nicht auf eine demokratisch und machtteilig organisierte Neuordnung zielt oder der Gegenentwurf nicht demokratisch und machtteilig konzipiert ist. Nichtstaatlicher Terrorismus kann vor diesem Hintergrund als in normativer Hinsicht illegitime und deshalb die empirische Legitimation durch die Zustimmung der Mehrheit mutmaßlich verfehlende, aber dennoch fortgeführte (sprich zeitlich und in der Folge auch hinsichtlich Qualität, Opfern, Zielen und im Falle des ,,islamistischen Terrorismus“ auch räumlich) entgrenzte politische Gewalt konzipiert werden.

Für den mutmaßlichen Zusammenhang der empirischen und der normativen Legitimation politischer Gewalt, die auf die Errichtung einer neuen Herrschaft zielt, und ihre Entgrenzung (hier vor allem hinsichtlich Zeit und Qualität) infolge mangelnder Legitimation scheinen Machiavellis Überlegungen zum neuen Fürsten eine ergiebige Quelle zu sein, gewissermaßen eine Schrift über Staatsterror avant la lettre. Machiavellis Principe ist ein Ratgeber für einen neuen Fürsten, der einen 
durch Gesetzlosigkeit gekennzeichneten Zustand ${ }^{19}$ unter Einsatz von Gewalt in eine Gesetzesherrschaft überführen soll. Die Parallele zur Unterscheidung zwischen gewaltsamem Widerstand und Terrorismus findet sich in der Unterscheidung zwischen dem neuen Fürst als Gründer einer Gesetzesherrschaft und dem Tyrann als Vertreter des Staatsterrors. ${ }^{20}$ Der Unterschied zwischen neuem Fürst und Tyrann liegt für Machiavelli ausdrücklich nicht in den verwendeten, auch gewaltsamen Mitteln, sondern in der Notwendigkeit bzw. Freiwilligkeit der Wahl dieser Mittel und damit zugleich der Dauer der Anwendung politischer Gewalt, die sich aus ihren Zielen erklärt (vgl. Höntzsch 2015a, S. 218-222).

Auch derjenige Alleinherrscher, der eine Gesetzesherrschaft (sprich legitime Herrschaft) errichten will, muss aufgrund des gesetzlosen Zustands Macht notfalls mit tyrannischen Mitteln erwerben: So war etwa Romulus' Brudermord in Machiavellis Augen gerechtfertigt, er dient im Principe wie in den Discorsi (in denen Machiavelli für eine republikanische Regierung votiert) als Vorbild, denn es handelt sich um eine Handlung, ,die er begangen hat, um ein Reich zu gründen oder einen Freistaat zu konstituieren. Spricht auch die Tat gegen ihn, so entschuldigt ihn doch der Erfolg“ (Machiavelli 1977, S. 36). Die Legitimation der Gewaltanwendung erweist sich empirisch erst nachträglich, bestätigt aber in Machiavellis Augen nur die normative Legitimation durch die Gemeinwohlorientierung der angestrebten Ordnung: „Daß aber Romulus wegen der Ermordung seines Bruders und seines Mitregenten $\mathrm{zu}$ entschuldigen ist und daß der Beweggrund seines Handelns das allgemeine Wohl und nicht eigensüchtige Herrschsucht war, beweist die sofortige Einsetzung eines Senats, mit dem er sich beriet und nach dessen Gutachten er seine Entscheidungen fällte“ (Machiavelli 1977, S. 37; Herv. d. Verf.). Die Orientierung am Gemeinwohl erweist sich, so lässt sich das verstehen, ex post darin, dass er die erworbene Macht teilt, anstatt absolute Macht auszuüben und also willkürlich zu herrschen, dass er also den gesetzlosen Zustand nicht in anderer Form, in Form einer Willkürherrschaft, fortführt, sondern beendet. Der neue Fürst handelt, insofern er dazu verdammt ist, nach den Regeln des Machterwerbs in einem gesetzlosen Zustand zu agieren, zwar wie ein Tyrann (er verwendet tyrannische Mittel); zum Tyrannen aber wird er erst in dem Moment, in dem er ohne Notwendigkeit bzw. ohne Blick auf das Gemeinwohl, sprich nur zum eigenen Nutzen, zur Befriedung des eigenen Ehrgeizes tyrannisch weiter regiert - ohne Notwendigkeit, weil er die erworbene Macht in eine Gesetzesherrschaft, die den Herrscher wie die Beherrschten gleichermaßen dem Gesetz unterwirft, überführen könnte. Das heißt während tyrannische, gewaltsame Mittel für Machiavelli zum Machterwerb in einem gesetzlosen Zustand durchaus legitim, weil für die Errichtung einer legitimen Herrschaft notwendig sind, sind sie es nicht, sobald die Macht gesichert ist und durch die Einführung einer Gesetzesherrschaft erhalten werden kann. Die Tyrannei

\footnotetext{
19 Machiavelli sieht Italien in einem „Endstadium“, „,unterdrückter als die Juden [...], geknechteter als die Perser, zerrissener als die Athener; ohne Führer, ohne gesetzliche Ordnung, geschlagen, geplündert, zerfleischt und von Feinden überrannt“ (Machiavelli 2001, S. 199-201).

20 Auch bei Machiavelli spielt hier interessanterweise die Erzeugung von Furcht eine wesentliche Rolle (Machiavelli 2001, Kap. XVII).
} 
ist so verstanden nichts anderes als die willentliche Perpetuierung, sprich zeitliche Entgrenzung, politischer Gewalt.

Unmittelbar vergleichbar sind Machiavellis Überlegungen im vorliegenden $\mathrm{Zu}$ sammenhang nur mit Fällen, in denen die politische Gewalt einer Minderheit vor dem Hintergrund fragiler oder zerfallender staatlicher Strukturen unmittelbar zum Machterwerb führt, wie im Falle des sogenannten „Islamischen Staates“, der sich in Anschluss an Machiavelli als tyrannische Herrschaft kennzeichnen lässt, insofern er zum Machterhalt am Einsatz von Gewalt festhält. Doch auch wenn die von einer oppositionellen Minderheit ausgehende politische Gewalt sich gegen eine etablierte, als willkürlich erachtete staatliche Gewalt wendet und so, anders als im Falle des neuen Fürsten, der vor dem Hintergrund von Gesetzlosigkeit handelt, nur über die Mobilisierung der Massen zum Machterwerb als Voraussetzung der Einführung einer legitimen (nicht willkürlichen) Herrschaft führt, lassen sich Machiavellis Überlegungen doch auch für die Unterscheidung zwischen gewaltsamem Widerstand und Terrorismus nutzbar machen: So gilt auch politische Gewalt gegen eine willkürliche Herrschaft zum Zwecke des Machterwerbs (bzw. zunächst der Befreiung) als legitimes Mittel, auch ihre Legitimation erfolgt aber im politischen Kampf um die Macht erst ex post; der Maßstab für die normative Legitimation bleibt derselbe, allerdings in hypothetischer Form, also nicht, wie im Fall des neuen Fürsten, die Frage, ob dem gewaltsamen Machterwerb eine Gesetzesherrschaft - bzw. eine Herrschaft, die Herrscher und Herrschende gleichermaßen an gleiches Recht bindet - folgt, sondern anhand der Frage, ob der Befreiung (und dem Machterwerb) ein Prozess der Neuordnung (und eine Herrschaft), die den Grund für den Widerstand - die Befreiung von Willkürherrschaft - in machtteilige, demokratische Verfahren übersetzt, folgen soll.

Die empirische Legitimation von durch eine Minderheit verübter (illegaler politischer) Gewalt, die sich ex post an der Zustimmung der Mehrheit bemisst, lässt sich so an die normative Legitimation rückbinden. Parallel zu Machiavellis Überlegungen lässt sich die These aufstellen, dass die Gewaltakteure die notwendige Zustimmung erlagen, wo sie eine demokratische und machtteilige Ordnung anstreben (was oft nur implizit der Fall ist, sprich, wo keine konkreten Ziele formuliert werden, nur ex negativo durch die Begründung des Widerstands gegeben ist), dagegen verfehlen, wo sie eine willkürliche Herrschaft anstreben. Das schließt nicht aus, dass empirisch auch Gruppen, die eine willkürliche Herrschaft anstreben, hinsichtlich der Massenmobilisierung erfolgreich sein können, die Vermutung liegt aber nahe, dass sie ihre Ziele zunächst verschleiern, jedenfalls aber aus ihrer Perspektive der Zustimmung der Massen zwar für den Machterwerb, nicht aber für die Legitimation der Herrschaft bedürfen. Ein Indikator für nichtstaatliche terroristische Gewalt scheint so bereits das Vorhandensein einer konkreten Vorstellung hinsichtlich der angestrebten Ordnung zu sein, insofern die Massen (als Träger der Revolution) hier von der Entscheidung über die politische Zukunft ausgeschlossen und gewissermaßen von der Minderheit (als Initiatoren des Umsturzes) zum Zwecke des eigenen Machterwerbs instrumentalisiert werden, weil die Gewaltmittel nicht ausreichen, um durch einen Staatsstreich unmittelbar an die Macht zu gelangen.

Terroristen stehen dabei nicht gleichermaßen vor der Wahl, wie der neue Fürst, der zum Tyrannen wird, weil er die im Moment des Machterwerbs notwendig ab- 
solute Macht nicht teilen will; sie perpetuieren die Gewalt, weil es ihnen allererst misslingt, Macht zu erwerben, weil sie die Mehrheit von der Legitimation ihres Anliegens nicht überzeugen und sie folglich nicht mobilisieren können. Während Machiavelli versucht, auf den Herrscher einzuwirken, weil es hier von dessen Entscheidung abhängt, ob er die gewaltsam erlangte Macht teilt oder absolut setzt, ist politische Gewalt, insofern sie sich gegen eine etablierte Herrschaft richtet, zum Erwerb der Macht auf die Zustimmung einer Mehrheit angewiesen. Wo die Akteure an der politischen Gewalt festhalten, sie also perpetuieren, obwohl bzw. weil die empirische Legitimation durch die Massen ausbleibt, wird sie notwendig ihre Gestalt ändern. Die in den gängigen Terror(ismus)definitionen herangezogenen Merkmale terroristischer Gewalt, können so als Entgrenzung infolge der ausbleibenden empirischen Legitimation verstanden werden. Nicht nur die Perpetuierung (als zeitliche Entgrenzung), auch die räumliche Entgrenzung im Falle des ,islamistischen Terrorismus“, die Ausweitung auf zivile Opfer (statt Beschränkung der Gewalt auf Funktionsträger), die Entgrenzung hinsichtlich der Qualität der Gewalt und auch der Ziele, in dem Sinne, dass Gewalt zum Selbstzweck wird, ist so verstanden Folge der Missachtung der verweigerten Legitimation bzw. Ausdruck der Ablehnung der Notwendigkeit der Legitimation durch die Mehrheit, was darauf verweist, dass keine machtteilige, demokratische Ordnung angestrebt wird. Die Entgrenzung politischer Gewalt kann so als empirischer Marker fehlender Legitimation gelten.

\section{Al-Qaida: Illegitime politische Gewalt}

Das Gesagte soll abschließend anhand von Selbstzeugnissen des sogenannten ,islamistischen Terrorismus“ plausibilisiert werden; genau genommen soll gezeigt werden, dass die verübte Gewalt hier von den Akteuren selbst als Widerstand gerechtfertigt wird und in der Folge - gemessen an der Rechtfertigung gewaltsamen Widerstands durch das Ziel der Befreiung von Willkürherrschaft - als illegitim und damit als Terrorismus gekennzeichnet werden kann. Vor allem letzteres kann hier nur angedeutet werden, unabhängig von den folgenden Ausführungen aber erscheint es wünschenswert, im Rahmen der Auseinandersetzung mit dem Phänomen des Terrorismus stärker die verfügbaren Quellen und damit die Perspektive der handelnden Akteure einzubeziehen. Als Textgrundlage soll im Folgenden Ayman al-Zawahiris Knights Under the Prophet's Banner (2006) und ergänzend Aussagen Osama bin Ladens dienen, die als wichtige Führungsfiguren und Vordenker von al-Qaida gelten (vgl. Kepel 2006).

Die Zielsetzung des sogenannten ,,islamistischen Terrorismus“ von al-Qaida entspricht zunächst der klassischen Zielsetzung gewaltsamen Widerstands gegen Willkürherrschaft. So hält Guido Steinberg (2005) mit Blick auf den ,islamistischen Terrorismus“ fest, dass er sich ,,in erster Linie als Reaktion auf die Herausforderungen der Moderne [entwickelte], meist als Protestbewegung gegen die eigenen als ,tyrannisch' wahrgenommenen Regierungen, die für sozioökonomische Probleme, kulturelle Entfremdung und politische Ohnmacht verantwortlich gemacht werden“" (S. 17; Herv. d. Verf.). Ähnlich konstatiert Fawaz A. Gerges in The Far Enemy: Why Jihad Went Global (2005): „Until the mid-1990s jihadist theory and practice focused 
almost exclusively on the domestic agenda and the need to replace the state of kufr (disbelief or rejection of divine guidance) with God's governance or sovereignty“ (S. 44; Herv. d. Verf.). Auch bei Zawahiri selbst wird deutlich, dass die politische Gewalt sich zu Beginn und in erster Linie gegen die nationalen - als gottlos verstandenen - Regierungen richtet. Zawahiri hält mit Blick auf den Mord an alSadat fest, ,that changing the regime, which had departed from Islam, became the central idea that preoccupied the Islamists, who rejected partial reform programs, patch-up jobs, and the attempts to beautify the ugly face of the regime with some reformatory measures" (Al-Zawahiri 2006 [2001], S. 61; Herv. d. Verf.).

Bei Zawahiri sind zentrale Momente der klassischen Rechtfertigung von Widerstand angesprochen: zum einen die Rechtfertigung der politischen Gewalt als „Widerstand“ gegen „Tyrannei“ (Zawahiri 2006, S. 109-110); genau genommen gegen eine gegen göttliches Recht verstoßende und deshalb als illegitim und willkürlich erachtete Regierung (bzw. später auch ihrer internationalen Unterstützer), sodass die Legitimation durch höheres, göttliches Recht erfolgt: „we must acknowledge that the west, led by the United States, which is under the influence of the Jews, does not know the language of ethics, morality, and legitimate rights. They only know the language of interests backed by brute military force" (Zawahiri 2006, S. 199; Herv. d. Verf.). Zum anderen wird hier die Abkehr von legalen Mitteln aufgrund ihrer Ineffektivität thematisiert: „The events proved the idea of work through material laws, submission to the secular consitution imposed by referendums, and recognition of the legitimacy of the government had become worn-out ideas in the minds of the Islamists" (Zawahiri 2006, S. 62). Die eigene Gewalt wird wenig überraschend und wiederum der klassischen Rechtfertigung von Widerstandshandlungen entsprechend als reaktiv gekennzeichnet. Die Schuld für die Gewalt trägt nicht al-Qaida, es handelt sich, in den Augen der Akteure, vielmehr um Gegengewalt. In diesem Sinne hält Osama bin Laden fest: ,,Vor diesem Hintergrund sind die Ereignisse vom 11. September geschehen als eine Erwiderung auf diese gewaltigen Ungerechtigkeiten, denn kann man dem einen Vorwurf machen, der sich nur verteidigt? Ist es auch Terrorismus, wenn man sich verteidigt und den Unterdrücker bestraft?" (Bin Laden 2006 [30. Oktober 2004], S. 131-132). Die westliche Perspektive verkehrend stellt Osama bin Laden in diesem Zusammenhang fest: „Der Staatsterror heißt Freiheit und Demokratie, und der Widerstand heißt Terrorismus und Opposition“ (Bin Laden 2006, S. 131).

Bei Zawahiri wird zugleich die enge Verbindung zwischen Widerstand bzw. Terrorismus und Revolution deutlich, wenn er als Ziel den Regimewechsel nennt: ,changing the regime, which had departed from Islam“. Hier sind beide Ziele illegaler politischer Gewalt angesprochen: Die Beseitigung einer als unrechtmäßig erachteten Herrschaft (,which had departed from Islam“) und das revolutionäre Element des Regimewechsels (,changing the regime“). Die revolutionäre Terminologie zeigt sich dabei insbesondere in der Unterscheidung von „Elite“ und „Masse“, die es zu mobilisieren gilt: „The jihad movement must come closer to the masses, defend their honor, fend off injustice, and lead them to the path of guidance and victory. [...] It must be extremely careful not to get isolated from its nation or engage the government in the battle of the elite against the authority“" (Al-Zawahiri 2006 [2001], S. 208-209). Zawahiri problematisiert die fehlende Unterstuitzung durch die Massen 
allerdings weniger aus legitimatorischen - aus seiner Sicht durchaus konsequent, weil als Legitimation die eigene als wahre Auslegung des göttlichen Rechts gilt denn aus agitatorischen Gründen:

In the absence of this popular support, the Islamic mujahed movement would be crushed in the shadows, far from the masses who are distracted or fearful, and the struggle between the Jihadist elite and the arrogant authorities would be confined to prison dungeons far from the public and the light of the day. This is precisely what the secular, apostate forces that are controlling our countries are striving for. These forces don't desire to wipe out the mujahed Islamic movement, rather they are stealthily striving to separate it from the misguided or frightened Muslim masses (Al-Zawahiri 2006, S. 258).

In dem hier zum Ausdruck kommenden instrumentellen Verständnis der Rolle der Akteure des Umsturzes, den ,fehlgeleiteten Massen“, die von der Elite auf „,den Pfad der Rechtleitung geführt" werden sollen, deutet sich an, dass weder der Prozess der Neuordnung noch die angestrebte Ordnung dem der Rechtfertigung von Widerstand als Befreiung von Willkürherrschaft inhärenten Maßstab entsprechen soll.

Der hier vorgeschlagene Maßstab kann sich der Perspektivität selbst nicht völlig entziehen, insofern er Widerstand aus einer westlich-liberalen Tradition heraus erklärt. Da die Akteure des ,islamistischen Terrorismus“ sich aber selbst der Rechtfertigungslogiken des klassischen Widerstandsdenkens bedienen, lässt sich ihnen ein Widerspruch nachweisen, insofern al-Qaida zwar erklärtermaßen (und - mit Blick auf die erste Stufe normativer Legitimation - u. U. auch berechtigterweise) Widerstand gegen eine als willkürlich erachtete Herrschaft leistet, aber nicht der in dieser Begründung ex negativo enthaltenen Forderung Folge leistet. Zwar wird die gleiche Bindung der Herrschenden wie der Beherrschten an göttliches Recht selbstverständlich postuliert, doch hinsichtlich der Auslegung der Rechtsquelle besteht ebenso selbstverständlich keine Gleichheit. Über die Zeit nach dem Abzug der Amerikaner und der Errichtung einer ,,governing authority“ hält Zawahiri fest:

Indeed, it's imperative that, in addition to force, there be an appeasement of Muslims and a sharing with them in governance and in the Shura council and in promulgating what is allowed and what is not allowed. [...] this must be achieved through the people of the Shura and who possess authority to determine issues and make them binding, and who are endowed with the qualifications for working in Sharia law (Al-Zawahiri 2006, S. 260).

Die Massen sollen zwar, so empfiehlt es Zawahiri, besser eingebunden werden (um nicht den Fehler der Taliban zu wiederholen, denen das afghanische Volk aufgrund mangelnder Partizipationsmöglichkeiten feindlich gesinnt war [vgl. Al-Zawahiri 2006, S. 262]), doch das islamische Recht, an das Herrschende und Beherrschte gleichermaßen gebunden sein sollen, ist - und hier lässt sich eine Parallele zur Begründung von Herrschaft und Widerstand im Rahmen des mittelalterlichen Herrschaftsvertragsdenkens ziehen - ein durch eine dazu berufene, dem göttlichen Recht kundige Minderheit ausgelegtes und in der Folge ungleiches Recht; worauf die Massen als Träger der Revolution keinen Einfluss haben, dem sie sich vielmehr fügen 
müssen. ${ }^{21}$ So ist zwar in den Statuten von al-Qaida, die sich als Vorschein auf die angestrebte Ordnung lesen lassen, auch eine Amtsenthebung des Anführers (Emirs) vorgesehen, allerdings soll diese - und hier ließe sich wiederum eine Parallele zum systeminternen Widerstand im Herrschaftsvertragsdenken ziehen - durch die Shura (nicht durch die zur Gesellschaft vereinten Individuen) erfolgen. ${ }^{22}$ Der Prozess der Neuordnung wie auch die angestrebte Neuordnung selbst widersprechen so gleichermaßen dem Ziel legitimer politischer Gewalt: der Befreiung von Willkürherrschaft durch die gleiche Bindung der Herrschenden und Beherrschten an gleiches Recht. Die räumliche Entgrenzung der Gewalt in Form der Ausweitung auf den ,fernen Feind" kann vor dem Hintergrund des Gesagten ebenso wie die zeitliche Entgrenzung in Form der Perpetuierung der Gewalt und die Entgrenzung hinsichtlich der Qualität, Opfer und Ziele der angewandten Gewalt als Marker der fehlenden Legitimation bzw. als Folge der empirisch nicht erfolgten Legitimation durch die Massen, die auf die fehlende normative Legitimation verweist, verstanden werden.

\section{Fazit}

Ziel der vorgeschlagenen Konzeption von Terrorismus ist es, die dem Begriff inhärente Kennzeichnung als illegitime Gewalt ernst zu nehmen, die durch ihn verübte illegale politische Gewalt aber nicht an den notwendig umstrittenen moralischen Überzeugungen der Gewaltakteure, sondern anhand eines wissenschaftlichen Maßstabes als illegitim zu kennzeichnen; genaugenommen, sofern Gewaltakteure die von ihnen verübte illegale (und als solche legitimationsbedürftige) politische Gewalt selbst notwendig als legitim ansehen müssen, anhand der klassischen Rechtfertigung gewaltsamen Widerstands: der Befreiung von Willkürherrschaft durch die gleiche Bindung der Herrschenden und Beherrschten an gleiches Recht. Eine solche Konzeption stellt gängige wissenschaftliche Terror(ismus)definitionen der Sache nicht infrage, sie fundiert diese vielmehr. Sie versteht die dort herausgearbeiteten Besonderheiten der durch Terroristen verübten Gewalt als Folge fehlender Legitimation und die Analyse der Mittel folglich der Frage nach den Zielen politischer Gewalt nachgeordnet. Die oftmals zur Definition terroristischer Gewalt herangezogenen Merkmale können so als Merkmale einer aufgrund ausbleibender empirischer Legitimation durch die Mehrheit, die auf die fehlende normative Legitimation verweist, auf Dauer gestellten, sprich zeitlich und in der Folge auch hinsichtlich ihrer Qualität, Opfer und Ziele (und im Falle des ,,islamistischen Terrorismus“ auch räumlich) entgrenzten Gewalt verstanden werden.

\footnotetext{
21 Dies versteht al-Qaida als religiöse Pflicht. So heißt es in der „Constitutional Charter“ von al-Qaida aus dem Jahr 2002: „The religious lawful duty, such as al-Jihad and the obedience to higher authorities, is every Muslim's duty by religious law“ (S. 4; Herv. d. Verf.); abrufbar unter: https://www.ctc.usma.edu/wpcontent/uploads/2013/10/Al-Qaida-Constitutional-Charter-Translation.pdf. Zugegriffen: 9. Oktober 2020.

22 Als deren Befugnis wird in „Al-Qaida’s Structure and Bylaws“ aus dem Jahr 2002 angeführt: ,dismiss the Emir thief he deviates from Shari'ah to the pint where he must be dismissed or when he loses his competency to be Emir and replace him with a new Emir“ (S. 13); abrufbar unter: https://www.ctc.usma. edu/wp-content/uploads/2013/10/Al-Qaida-Constitutional-Charter-Translation.pdf. Zugegriffen: 9. Oktober 2020 .
} 
Die vorgeschlagene Konzeption stellt dabei nicht nur einen wissenschaftlichen Maßstab zur Bezeichnung politischer Gewalt als Terrorismus bereit, zugleich ermöglicht der mehrstufige Maßstab einen differenzierten Blick auf konkrete Phänomene: So lässt sich trennen zwischen der Berechtigung des Ziels der Befreiung von Willkürherrschaft - die, so sie (wie das oftmals der Fall ist) gegeben ist, zugleich der Grund für das Mobilisierungs- und Radikalisierungspotenzial terroristischer Gruppen ist - und der Frage nach der Übersetzung dieses Anliegens in den Prozess der Neuordnung sowie die angestrebte Ordnung. Eine pauschale Verurteilung politischer Gewalt als Terrorismus wird folglich, dort wo sie dem Kampf gegen herrschaftliche Willkür die Berechtigung abspricht und dadurch ein korruptes Regime stützt, terroristischen Gruppen in die Hände spielen; eine Differenzierung scheint hier dringend erforderlich, um demokratische Kräfte zu stärken. Vor dem Hintergrund einer solchen Differenzierung wird zugleich deutlich, dass eine erfolgreiche Revolution bzw. zunächst eine gelingende Befreiung zugleich das beste Gegenmittel gegen den Terrorismus ist: „When dictators such as Mubarak fall due to pressure from prodemocracy protesters [...] al Qaeda loses one of its best recruiting pitches: the repression Arab governments inflict on their citizens. The rise of less repressive leaders would deprive al Qaeda propagandists of this valuable argument" (Byman 2011, S. 49). In diesem Fall könnte auch terroristische Gewalt in einer demokratisch und machtteilig organisierten Neuordnung aufgehen bzw. von dieser im Keim erstickt werden.

Danksagung Ich danke den anonymen Gutachter*innen für die konstruktive Kritik und hilfreiche Anmerkungen.

Funding Open Access funding enabled and organized by Projekt DEAL.

Open Access Dieser Artikel wird unter der Creative Commons Namensnennung 4.0 International Lizenz veröffentlicht, welche die Nutzung, Vervielfältigung, Bearbeitung, Verbreitung und Wiedergabe in jeglichem Medium und Format erlaubt, sofern Sie den/die ursprünglichen Autor(en) und die Quelle ordnungsgemäß nennen, einen Link zur Creative Commons Lizenz beifügen und angeben, ob Änderungen vorgenommen wurden.

Die in diesem Artikel enthaltenen Bilder und sonstiges Drittmaterial unterliegen ebenfalls der genannten Creative Commons Lizenz, sofern sich aus der Abbildungslegende nichts anderes ergibt. Sofern das betreffende Material nicht unter der genannten Creative Commons Lizenz steht und die betreffende Handlung nicht nach gesetzlichen Vorschriften erlaubt ist, ist für die oben aufgeführten Weiterverwendungen des Materials die Einwilligung des jeweiligen Rechteinhabers einzuholen.

Weitere Details zur Lizenz entnehmen Sie bitte der Lizenzinformation auf http://creativecommons.org/ licenses/by/4.0/deed.de.

\section{Literatur}

Al-Zawahiri, Ayman. 2006. Knights under the prophet's banner. In His own words. A translation of the writings of Dr. Ayman al Zawahiri, Hrsg. Laura Mansfield, 17-225. N.N.: TLG Publications.

Aquin, Thomas von. 1933ff. Summa Theologiae. Heidelberg, Graz: Kerle, Styria. 37 Bde, lat.-dt., Deutsche Thomas-Ausgabe.

Aquin, Thomas von. 2004. Über die Herrschaft des Fürsten. Stuttgart: Reclam.

Arendt, Hannah. 2000. Ziviler Ungehorsam. In In der Gegenwart. Übungen im politischen Denken II, Hrsg. Hannah Arendt, 283-321. München/Zürich: Piper. 
Arendt, Hannah. 2012. Revolution und Freiheit. In Zwischen Vergangenheit und Zukunft. Übungen im politischen Denken I, Hrsg. Hannah Arendt, 227-251. München/Zürich: Piper.

Aristoteles. 1996. Politik, Buch IV-VI. Werke in deutscher Übersetzung, Bd. 9. Berlin: Akademie Verlag. übers. und eingel. v. Eckart Schütrumpf, erläutert v. dems. und Hans-Joachim Gehrke.

Bakunin, Michail. 1972. Die Prinzipien der Revolution (1869). In Staatlichkeit und Anarchie und andere Schriften, Hrsg. Horst Stuke, 100-105. Frankfurt a. M.: Ullstein.

Ballestrem, Karl Graf von. 1983. Vertragstheoretische Ansätze in der politischen Philosophie. Zeitschrift für Politik 30(1):1-17.

Bin Laden, Osama. 2006. Botschaft an das amerikanische Volk [30. Oktober 2004]. In Al-Qaida. Texte des Terrors, Hrsg. Gilles Kepel, Jean-Pierre Milelli, 129-136. München, Zürich: Piper.

Brutus, Stephanus Junius. 1968. Strafgericht gegen die Tyrannen oder: Die legitime Macht des Fürsten über das Volk und des Volkes über den Fürsten. In Beza, Brutus, Hotman. Calvinistische Monarchomachen, Hrsg. Jürgen Dennert, 61-202. Köln: Opladen.

Byman, Daniel. 2011. After the revolution: how secular uprisings could help (or hurt) Jihadists. Foreign Affairs 90(3):48-54.

Celikates, Robin. 2010. Ziviler Ungehorsam und radikale Demokratie. In Das Politische und die Politik, Hrsg. Thomas Bedorf, Kurt Röttgers, 274-300. Frankfurt a. M.: Suhrkamp.

Celikates, Robin, und Frauke Höntzsch. 2019. Editorial der Gastherausgeber. Zeitschrift für Politische Theorie 2019(1):3-8.

Cicero, Marcus Tullius. 1979. De re publica/Vom Gemeinwesen. Stuttgart: Reclam. Lat./Dt., übers. und hrsg. v. Karl Büchner.

Colgan, Jeff. 2012. Measuring revolution. Conflict Management and Peace Science 29(4):444-467.

Crenshaw, Martha. 1972. The concept of revolutionary terrorism. The Journal of Conflict Resolution 16(3):383-396.

Crenshaw, Martha. 1983. Introduction. In Terrorism, legitimacy and power: the consequences of political violence, Hrsg. Martha Crenshaw, 1-38. Middletown: Wesleyan University Press.

Daase, Christopher. 2013. Terrorismus. In Handbuch Politische Gewalt. Formen, Ursachen, Legitimation, Begrenzung, Hrsg. Birgit Enzmann, 335-348. Wiesbaden: Springer.

Daase, Christopher. 2014. Was ist Widerstand? Zum Wandel von Opposition und Dissidenz. Aus Politik und Zeitgeschichte 27:3-9.

Della Porta, Donatella. 1995. Social movements, political violence, and the state. Cambridge: Cambridge University Press.

Della Porta, Donatella. 2013. Clandestine political violence. Cambridge: Cambridge University Press.

Della Porta, Donatella, und Sidney Tarrow. 1986. Unwanted children: political violence and the cycle of protest in Italy, 1966-1973. European Journal of Political Research 14:607-632.

Dennert, Jürgen. 1968. Einleitung. In Beza, Brutus, Hotman. Calvinistische Monarchomachen, Hrsg. Jürgen Dennert, IX-LXXV. Köln: Opladen.

Dunn, John. 1984. The concept of trust in the politics of John Locke. In Philosophy in history. Essays on the historiography of philosophy, Hrsg. Richard Rorty, J.B. Schneewind, und Quentin Skinner, 279-301. Cambridge: University Press.

Enzmann, Birgit. 2013a. Politische Gewalt. Formen, Hintergründe, Überwindbarkeit. In Handbuch Politische Gewalt. Formen, Ursachen, Legitimation, Begrenzung, Hrsg. Birgit Enzmann, 43-66. Wiesbaden: Springer.

Enzmann, Birgit. 2013b. Revolution. In Handbuch Politische Gewalt. Formen, Ursachen, Legitimation, Begrenzung, Hrsg. Birgit Enzmann, 205-230. Wiesbaden: Springer.

Finlay, Christopher J. 2015. Terrorism and the right to resist. A theory of jus revolutionary war. Cambridge: Cambridge University Press.

Gerges, Fawaz A. 2005. The far enemy. Why Jihad went global. Cambridge: Cambridge University Press.

Goldstone, Jack A. 2001. Toward a fourth generation of revolutionary theory. Annual Review of Political Science 4(1):139-187.

Habermas, Jürgen. 1983. Ziviler Ungehorsam. Testfall für den demokratischen Rechtsstaat. Wider den autoritären Legalismus in der BRD. In Ziviler Ungehorsam im Rechtsstaat, Hrsg. Peter Glotz, 29-53. Frankfurt a. M.: Suhrkamp.

Hardman, J.B.S. 1948. Terrorism. Encyclopaedia of the Social Sciences 14:575-580.

Höntzsch, Frauke. 2010. Gewaltentrennung und Widerstandsrecht. Komplementäre Konzepte zum Schutz von Leben, Freiheit und Besitz im politischen Denken von John Locke. In Der Staat des Liberalismus, Hrsg. Samuel Salzborn, 165-184. Baden-Baden: Nomos. 
Höntzsch, Frauke. 2015a. Machiavellis realistischer Fürstenspiegel. Il Principe als Appell an den Ehrgeiz. In Die literarische Formierung der politischen Moderne. Spätmittelalter und Renaissance in Italien, Hrsg. Oliver Hidalgo und Kai Nonnenmacher, 207-224. Wiesbaden: Springer VS.

Höntzsch, Frauke. 2015b. Für eine politikwissenschaftliche Ideengeschichte. In Neue Perspektiven der Ideengeschichte, Hrsg. Helmut Reinalter, 75-89. Innsbruck: University Press.

Jenkins, Brian. 1980. The study of terrorism. Definitional problems. https://www.rand.org/content/dam/ rand/pubs/papers/2006/P6563.pdf. Zugegriffen: 11. Okt. 2019.

Kepel, Gilles. 2006. Allgemeine Einführung. Der Kern von Al-Qaida. In Al-Qaida. Texte des Terrors, Hrsg. Gilles Kepel, Jean-Pierre Milelli, 13-21. Zürich: Piper.

Klautke, Jürgen-Burkhard. 1994. Recht auf Widerstand gegen die Obrigkeit? Eine systematisch-theologische Untersuchung zu den Bestreitungs- und Rechtfertigungsbemühungen von Gewaltanwendung gegen die weltliche Macht (bis zum 18. Jahrhundert). Bd. 1. Kampen: Kok.

Kydd, Andrew H., und Barbara F. Walter. 2006. The strategies of terrorism. International Security 31(1):49-80.

Laqueur, Walter. 1986. Reflections on terrorism. Foreign Affairs 65(1):86-100.

Laqueur, Walter. 1996. Postmodern terrorism. Foreign Affairs 75(5):24-36.

Laqueur, Walter. 1999. The new terrorism. Oxford: Oxford University Press.

Lewy, Guenter. 1960. Constitutionalism and statecraft during the golden age of Spain. A study of the political philosophy of Juan de Mariana. Genf: E. Droz.

Locke, John. 1988. Two treatises of government. Cambridge: Cambridge University Press. ed. by Peter Laslett.

Machiavelli, Niccolo. 1977. Discorsi. Gedanken über Politik und Staatsführung. Stuttgart: Kröner. übersetzt, eingeleitet und erläutert von Dr. Rudolf Zorn.

Machiavelli, Niccolo. 2001. Il Principe/Der Fürst. Stuttgart: Reclam. übersetzt und hrsg. von Philip Rippel.

Markovits, Daniel. 2005. Democratic disobedience. Yale Journal 114:1897-1952.

Marx, Karl. 1959. Sieg der Konterrevolution zu Wien [1848]. In MEW 5, Hrsg. Karl Marx, Friedrich Engels, 455-457. Berlin: Dietz.

Münkler, Herfried. 1995. Widerstandslehren. In Politische Theorien Lexikon der Politik, Bd. 1, Hrsg. Dieter Nohlen, Rainer-Olaf Schultze, 691-696. München: C. H. Beck.

Münkler, Herfried. 2004. Ältere und jüngere Formen des Terrorismus. In Herausforderung Terrorismus, Hrsg. Werner Weidenfeld, 29-43. Wiesbaden: VS.

Neumann, Peter R. 2009. Old and new terrorism. Late modernity, globalization and the transformation of political violance. Cambridge: Polity Press.

Rapoport, David. 2004. The four waves of terrorism. In Attacking terrorism: elements of a grand strategy, Hrsg. Audrey K. Cronin, James M. Ludes, 46-73. Washington, DC: Georgtown University Press.

Rawls, John. 1979. Eine Theorie der Gerechtigkeit. Frankfurt a. M.: Suhrkamp.

Reinares, Fernando. 2002. Terrorismus. In Internationales Handbuch der Gewaltforschung, Hrsg. Wilhelm Heitmeyer, John Hagan, 390-405. Wiesbaden: Westdeutscher Verlag.

Robespierre, Maximilien. 1989. Über die Grundsätze der revolutionären Regierung (1793). In Ausgewählte Texte, Hrsg. Maximilien de Robespierre, 562-581. Hamburg: Merlin.

Schmid, Alex P. 2013. The definition of terrorism. In The Routledge handbook of terrorism research, Hrsg. Alex P. Schmid, 39-98. London, New York: Routledge.

Schmid, Alex P., und A.J. Jongman. 1988. Political terrorism. A new guide to actors, authors, concepts, data bases, theories and literature. New Brundswick: Transaction Books.

Schneckener, Ulrich. 2015. Transnationaler Terrorismus. Charakter und Hintergründe des „neuen “Terrorismus, 3. Aufl., Frankfurt a. M.: Suhrkamp.

Schrader, Tobias. 2002. Terrorismus und das Problem seiner Definition. Krim 56:560-572.

Spindelböck, Josef. 1994. Aktives Widerstandsrecht. Die Problematik der sittlichen Legitimität von Gewalt in der Auseinandersetzung mit ungerechter staatlicher Macht. St. Ottilien: EOS.

Sprinzak, Ehud. 1991. The process of delegitimization: towards a likage theory of political terrorism. In Terrorism research and public policy, Hrsg. Clark McClauley, 50-68. London: Frank Cass.

Steinberg, Guido. 2005. Der nahe und der ferne Feind. Die Netzwerke des islamistischen Terrorismus. München: Beck.

Stüttler, Josef Anton. 1972. Das Widerstandsrecht und seine Rechtfertigungsversuche im Altertum und im frühen Christentum. In Widerstandsrecht, Hrsg. Arthur Kaufmann, 1-58. Darmstadt: Wissenschaftliche Buchgesellschaft.

Tarcov, Nathan. 1999. Locke's second treatise and ,the best fence against rebellion“. In Locke's moral, political and legal philosophy, Hrsg. John R. Milton, 198-217. Aldershot: Dartmouth. 
Thoreau, Henry David. 1966. Walden and civil disobedience. London: Norten Critcal Editions. hrsg. v. Thomas Owen.

Townshend, Charles. 2005. Terrorismus. Eine kurze Einführung. Stuttgart: Reclam.

von Aquin, Thomas. 2019. Sentenzenkommentar. http://www.corpusthomisticum.org/iopera.html. Zugegriffen: 9. Okt. 2020.

Waldmann, Peter. 2005. Terrorismus. Provokation der Macht, 2. Aufl., Hamburg: Murmann.

Weinberg, Leonard (Hrsg.). 1992. Political parties and terrorist groups. London: Frank Cass.

Weinberg, Leonhard, Ami Pedahzur, und Sivan Hirsch-Hoeffler. 2004. The challenges of conceptualizing terrorism. Terrorism and Political Violence 16(4):777-794.

Wiktorowicz, Quintan. 2004. Islamic activism: a social movement theory approach. Bloomington: Indiana University Press.

Wolf, Dieter, und Michael Zürn. 1995. Revolutionstheorien. In Politische Theorien Lexikon der Politik, Bd. 1, Hrsg. Dieter Nohlen, Rainer-Olaf Schultze, 552-561. München: Beck.

Wolzendorff, Kurt. 1968. Staatsrecht und Naturrecht in der Lehre vom Widerstandsrecht des Volkes gegen rechtswidrige Ausübung der Staatsgewalt. Aalen: Scientia. 